\title{
HIGH TIME RESOLUTION ASTROPHYSICS
}

XXVII Canary Islands Winter School of Astrophysics

\author{
TARIQ SHAHBAZ, \\ Instituto de Astrofísica de Canarias, Tenerife, Spain \\ Departamento de Astrofísica, Universidad de La Laguna, Tenerife, Spain \\ JORGE CASARES VELÁZQUEZ \\ Instituto de Astrofísica de Canarias, Tenerife, Spain \\ Departamento de Astrofísica, Universidad de La Laguna, Tenerife, Spain \\ TEODORO MUÑOZ DARIAS \\ Instituto de Astrofísica de Canarias, Tenerife, Spain \\ Departamento de Astrofísica, Universidad de La Laguna, Tenerife, Spain
}





\section{Contents}

1 Black-hole and neutron-star binaries

page 1

1.1 Introduction 1

1.2 Neutron-Star binaries 3

1.2.1 Evolution and subclasses

1.2.2 Energy spectra

1.2.3 Timing properties 8

1.3 Black-Hole binaries 12

1.3.1 States and state-transitions 12

1.3.2 Energy spectra

1.3.3 Timing properties 20

1.4 Comparison between NS and BH systems 26

1.4.1 Quiescent emission 27

1.4.2 X-ray bursts 27

1.4.3 Energy spectra 27

1.4.4 Time variability 29

1.4.5 Mass measurements from X-rays 32

1.5 Conclusions 38

1.6 Acknowledgements 38 



\title{
X-ray emission from black-hole and neutron-star binaries
}

\author{
TOMASO M. BELLONI
}

\begin{abstract}
In this chapter, I present the main X-ray observational characteristics of black-hole binaries and low magnetic field neutron-star binaries, concentrating on what can be considered similarities or differences, with particular emphasis on their fast-timing behaviour.
\end{abstract}

\subsection{Introduction}

The large amount of information that has become available in the past two decades on high-energy emission from accreting X-ray binaries, both in terms of number of observations and high-resolution high-sensitivity data, have given us a much more complete view of the emission properties from the inner regions of the accretion flow, close to the compact object. While it is now nearly impossible to review all observational aspects, in this chapter I will present an overview of the X-ray characteristics with a special emphasis on the comparison between Galactic neutron-star (NS) and black hole (BH) accreting systems. Since the strong dipolar magnetic field (B) in the 'standard' X-ray pulsars dominates the accretion flow and prevents the formation of nearly-equatorial accretion in the innermost few gravitational radii around the NS, I will concentrate on low-field NS objects, most of which are low-mass X-ray binaries (LMXB). As the size of the region inside the innermost stable circular orbit (ISCO) around a BH and the size of a NS are roughly comparable, one can expect that many properties of the accretion flow and its emission would be in common between the two classes of systems. However, the presence of a solid surface on a NS, which prevents advection of energy, must necessarily add another emission component, which can be very complex as a boundary layer is formed. Moreover, the dipolar 
magnetic field in a LMXB, estimated to be in the range $10^{8}-10^{9} \mathrm{G}$, is still not negligible and must alter the properties of accretion in the innermost regions, where its energy density is higher.

Optical observations of the companion star have now provided robust dynamical mass measurements (see Casares and Jonker, 2014, for a recent review). From the early days of X-ray astronomy, after it was realised that the presence of a $\mathrm{BH}$ and/or NS was the origin of the strong high-energy emission, we have tried to identify specific 'signatures' in the high-energy emission, which would allow us to identify unambiguously the nature of the compact object. While we know a few of these signatures for NSs, such as coherent pulsations (now detected in more than a dozen accreting LMXBs) and thermonuclear X-ray bursts, 'smoking guns' that reveal directly the presence of a $\mathrm{BH}$ are still elusive (see van der Klis, 1994 , for a review of the observational status of the comparison between NS and BH before $X M M$ Newton, Chandra and RossiXTE). This issue is strongly connected to the detection of effects due to General Relativity (GR) in the strong field regime as can only found in the proximity of a compact object, where the theory still needs to be tested. The recent direct detection of gravitational waves from coalescing BHs is of course one (Abbott et al. 2016), but the most precise measurements to date come from binary pulsars, which are still hundreds of thousands kilometres apart (see Burgay et al., 2014).

On the one hand, we are looking for a common observational scenario that can be interpreted within a single accretion model, since the accretion flow around BHs and weakly magnetised NSs is expected to be very similar. On the other hand, it is important to find crucial observables that allow a direct identification of the presence of a $\mathrm{BH}$, in the way that pulsations or thermonuclear X-ray bursts indicate the presence of a surface and therefore of a NS. These observables would also allow us to measure additional parameters predicted by $\mathrm{GR}$ such as $\mathrm{BH}$ spin, relativistic precessions and the presence of an ISCO (see Psaltis, 2008). There is considerable observational effort aimed at measuring BH spins through X-ray spectra (see Middleton, 2016), although these methods all need to assume a BH mass, which until now can only be measured through dynamical methods, i.e. indirectly. Although it is true that optical measures have become much more accurate in the past decades, the issue of absence of direct evidences does not depend on measurement accuracy and cannot be solved in that way. Along the way, we moved from the expression 'black hole candidate' to 'black hole binary' (which admittedly sounds much better) for dynamically-confirmed cases, relegating the former to systems without mass measurement, but the situation has not changed and the associated caveats remain. 
This cannot be considered a full review. Here I outline the main X-ray emission properties of accreting binaries containing a $\mathrm{BH}$ or a low-B neutron star, limiting myself to those that can be used to establish similarities or differences between classes. The focus is on timing properties, although spectral information is also discussed as discussing timing only would not allow to present a complete picture, necessary to understand these systems. Moreover, I will limit myself to the X-ray band. This means I will ignore huge parts of the field of the astrophysics of accretion onto stellar-mass compact objects, which would also require much more space than available. This text should be sufficient as an introduction and a companion to extricate oneself through the extensive literature in the field.

\subsection{Neutron-Star binaries}

\subsubsection{Evolution and subclasses}

Since the 1980s, NS LMXBs have been divided into classes, based on the timing and spectral characteristics. Since the spectral decomposition for these systems is complex (see below) and significant variability takes place on a time-scale of minutes to hours, the spectral variations are best followed through the use of colour-colour diagrams (CCDM), where two spectral 'colours' (ratios between detected counts in two energy bands) integrated over short time-scales (minutes) are plotted against each other. NS LMXBs trace very clear patterns on their CCDM, moving in a smooth way without major jumps in time (see figures 2.4 in van der Klis, 2006). Depending on the shape of the CCDM track and the associated timing properties, classes and subclasses were identified.

Six bright sources, all persistently at high X-ray flux, were called 'Z sources' as they trace a path roughly shaped as a letter Z $\mathrm{Z}$. Of course the precise shape of the path depends on the instrument and on the chosen energy bands; moreover, at different times the path can be observed to shift in the CCDM. The six sources are: Sco X-1, Cyg X-2, GX 340+0, GS 349+2, GX 5-1 and GX 17+2. A second subclass is that of 'atoll' sources, who owe their bizarre name to the fact that their CCDM looks like a tropical atoll. This class is much more populated, with dozens of systems known to date. In addition, there is a class of sources at low luminosity, which typically show type-I X-ray bursts, commonly referred to as 'low-luminosity bursters.' A unique system such as Cir X-1 has long been considered not to be classifiable in this scheme, although now it is added to the Z-source class.

The idea of following systems in the CCDM, which led to this colourful 

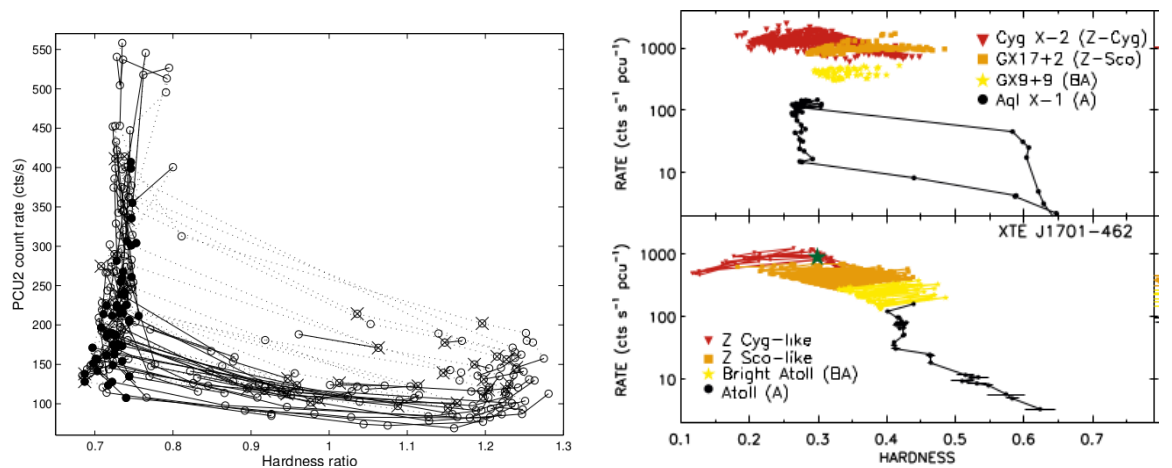

Figure 1.1 Left panel: HID of 4U 1636-53 from $R X T E$ data. Solid lines connect indicate movement to the right (hardening), dotted lines movement to the left (softening). (From Belloni et al., 2007b). Right panel: HID for the Z sources Cyg X-2 and GX 17+2, the atoll source source GX $9+9$ and the transient Aql X-1 (top), HID for the Z/atoll transient XTE J1701-462. (From Muñoz-Darias et al., 2014).

terminology, comes from the difficulty of disentangling spectral components in these systems. The presence of a number of spectral components that emit in a comparable energy band make it difficult to follow changes on short time-scales. The use of a purely observational tool such as the CCDM, although instrument-dependent, allows to track these changes very effectively. In addition, it was found that the properties of fast timing correlate rather precisely with the position on the CCDM. In analogy with $\mathrm{BH}$ binaries (BHBs), it is interesting to produce also a hardness-intensity diagram (HID), where a single hardness is plotted versus intensity, for Z sources and for transient and persistent atoll sources (see Figure1.1). One can see that atoll sources, both persistent (such as 4U 1636-53) and transient (such as AqlX-1), follow a hysteresis cycle in the HID. In the case of $4 \mathrm{U} 1636-53$, during RossiXTE observations the flux oscillated with a quasi-period of 30$40 \mathrm{~d}$, following a loop in the HID. Other systems are not so regular, but their behaviour is consistent (see figure 2 in Muñoz-Darias et al., 2014).

Until a decade ago, there was no agreement as to what the fundamental difference between $\mathrm{Z}$ and atoll sources was. Although it was suspected that accretion rate was responsible, the effect of a different value of the dipolar magnetic field of the NS could not be excluded (see Hasinger and van der Klis, 1989, for a review). Since the distances to these systems are in most cases not determined, it is difficult to measure directly their luminosity. Even for single $\mathrm{Z}$ sources, accretion rate was found not to be tracked by source 
intensity Hasinger and van der Klis (1989). In January 2006, a bright transient system appeared in the sky, XTE J1701-462 (Remillard et al., 2006a). It was soon realised that this was a transient Z-source (Homan et al. 2007). As the outburst progressed, the system passed through phases where it displayed CCDM shapes typical of all subclasses of $\mathrm{Z}$ sources, until after about $1.5 \mathrm{yr}$ the accretion rate dropped rapidly and before reaching quiescence the system went through a brief 'atoll' phase, showing clearly that the difference between classes can be ascribed solely to the effect of accretion rate (see Homan et al., 2010, see also Figure 1.1).

Working with a large sample of systems from the RossiXTE archive, Muñoz-Darias et al. (2014) have compared the HID properties of NS LMXBs and proposed a general scheme to put together different classes (and to compare them to BHBs, see below). Their unified diagram can be seen in Figure 1.2 , where a RMS-intensity diagram (RID) is shown [here hardness is replaced by fractional root-mean-squared (RMS) variability, which however correlates well with hardness, and luminosity rather than count rate is on the $\mathrm{Y}$ axis, necessary to compare different systems]. In this sketch, the connection between $\mathrm{Z}$ and atoll sources is clear and only associated to a difference in luminosity. In this diagram, the low-luminosity bursters live on bottom-right branch, always in the hard state and at low luminosity.

One state is missing in this description, namely the quiescent state of transients, when accretion rate is low and instruments such as the $R X T E / \mathrm{PCA}$ cannot detect X-ray emission. Most of the time, transients are in quiescence, at an accretion luminosity below $10^{33} \mathrm{erg} / \mathrm{s}$. While in the case of BHBs (see below), any X-ray emission observed in quiescence can only come from the accretion flow (with a possible contribution of the X-ray emission of the companion late-type star), NS LMXBs feature a hard surface. This has two practical effects: the first is that the energy from any residual low-level accretion must be released, even in case of inefficient accretion (see e.g. Menou et al., 1999; Kong et al., 2002). The second is that if accretion stops completely, after the effects of accretion have disappeared, the cooling curve of the NS can be measured (see e.g. Brown and Cumming, 2009). The presence of the surface emission makes the positioning on the HID not very relevant.

\subsubsection{Energy spectra}

The energy spectra of NS LMXBs are complex. The presence of the hard surface of the NS introduces an extra component that is in the same energy range than that (or those) from the accretion flow. As a result, two different spectral models have been proposed at the end of the 1980s, which 


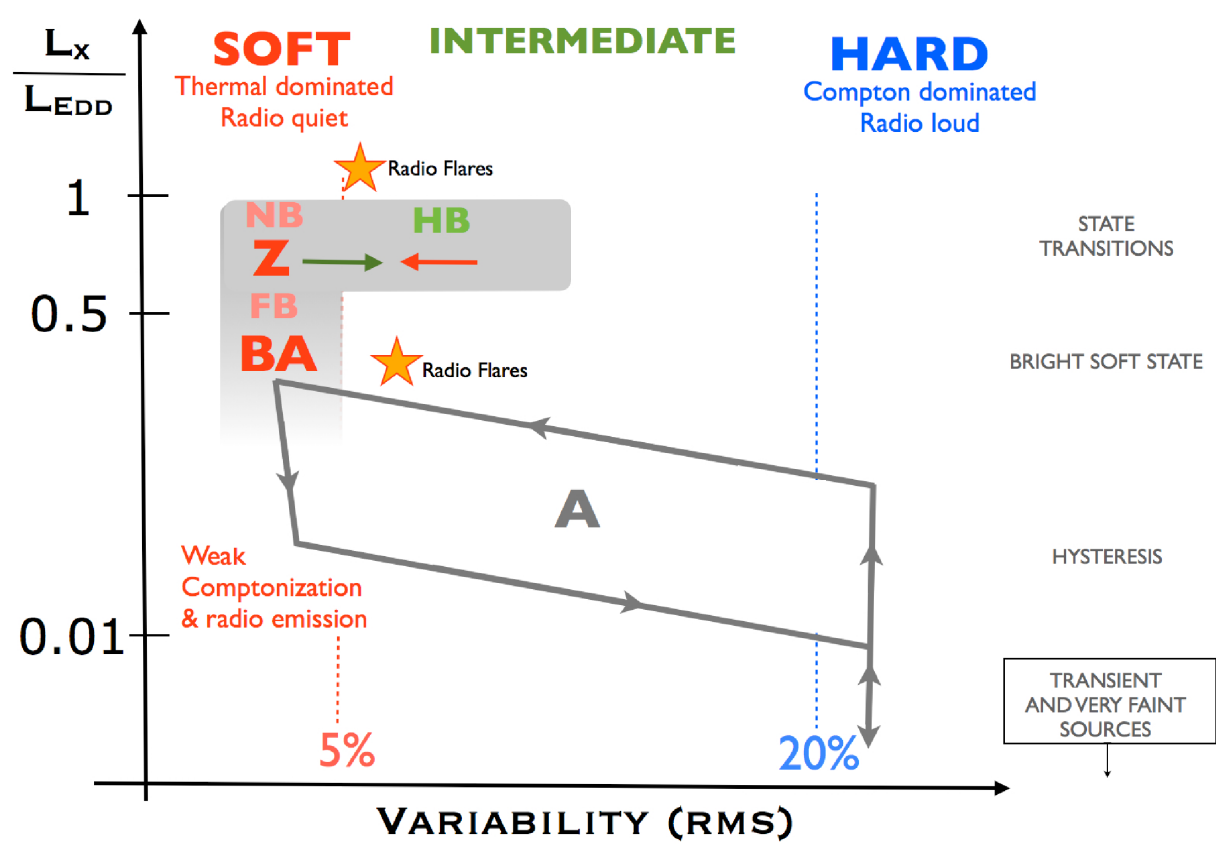

Figure 1.2 Schematic RMS-intensity diagram describing the position and behaviour of atoll (A), bright atoll (A) and Z (Z) sources. A HID has qualitatively the same shape. (From Muñoz-Darias et al., 2014).

have hardly been addressed until recent times, when a much larger number of spectra over different states have been accumulated, mostly by the RossiXTE satellite. As one can see from Figure 1.2, the observations indicate the presence of two main states, which need to be interpreted within a common framework. These states are connected by intermediate states, which add complications, but also allow a connection between hard and soft states. The two 'old time' models are traditionally referred to as the Western and the Eastern model. For the hard state, both models are compatibile and consist of a hard Comptonised component with an electron temperature of dozens of $\mathrm{keV}$, plus a softer thermal component with temperature $<1 \mathrm{keV}$ that can be modelled as a black-body (BB) or a disk-blackbody (DBB) (see e.g. Gierliński and Done, 2002). These spectra are analogous to those of BHBs in their hard state (see below) and as we will see their fast timing properties are also very similar to those of BHBs. For the soft state, the Eastern model (Mitsuda et al. 1989$)$ includes a DBB $(\mathrm{kT} \sim 0.5-2 \mathrm{keV})$ and a weakly Comptonised BB, while the Western model (White et al., 
1988) decomposes the spectra as a blackbody from the boundary layer and a Comptonised disk component.

Lin et al. (2007) analyzed a large number of RossiXTE observations, with which they could follow the spectral changes of two atoll systems across states and concluded that not only all combinations of a thermal and a nonthermal component are degenerate, but that it is impossible to reconcile observations in different states with the two models outlined above. They proposed a third, hybrid model: in the hard state the spectrum is deconvolved into a blackbody and a Comptonised component, like in the older models. In the soft state, a three-component model is needed, consisting of a DBB, a BB and a weakly Comptonised component. This model has the advantage to maintain the $\mathrm{L} \propto \mathrm{T}^{4}$ relation expected for a $\mathrm{DBB}$, to have a constant area for the BB component (corresponding to that of the boundary layer, around $15 \%$ that of the surface of the NS), and to have a Comptonised fraction compatible with that of BHBs. This work was extended to the transitional system XTE J1701-462 (Lin et al., 2009) and to the persistent Z source GX 17+2 (Lin et al., 2012), i.e. to systems at higher accretion rate. The conclusion was that accretion rate drives the secular changes in shape of the $\mathrm{Z}$ diagram, while the actual motion along the branches of the diagram takes place at constant accretion rate and are driven by other mechanisms such as change in the Comptonised fraction, transition to a slim disk solution and changes in the inner disk radius. All these results can be linked very effectively with the characteristics observed in BHBs, as shown below.

The availability of X-ray instruments with high energy resolution has allowed to detect and study additional components in the energy spectra of NS LMXBs, such as Compton reflection features (broad iron lines and continuum). Several systems have been studied (see e.g. Di Salvo et al., 2015, Degenaar et al., 2015, and references therein), which led to constraints to the inner disk structure and inclination. I will discuss an application of these models in combination to timing models for mass measurement below. Additional non-thermal components have been detected in $\mathrm{Z}$ sources in the form of hard spectral tails (see Migliari et al., 2007, and references therein). They can contribute up to $10 \%$ of the total flux and appear to be in general stronger in the HB (see Figure 1.2). The possible interpretation proposed range from non-thermal electrons in a corona or at the base of a jet (see Markoff et al., 2005) to bulk motion Comptonisation in a converging flow (Farinelli et al., 2008). 


\subsubsection{Timing properties}

The phenomenology of the observed features in the power density spectra (PDS) of NS LMXBs is very extensive (see van der Klis, 2006, for a review). Here I present the main points, in order to limit myself to the most basic picture, which can then be compared to BHBs.

Low frequencies. At low frequencies $(\leq 100 \mathrm{~Hz})$ the PDS of NS LMXBs are complex. In the hard state (see Figure 1.2), at least four zero-centered Lorentzian components are needed to model the variability (see the left panel in Figure 1.3 and the integrated fractional RMS is high, larger than $20 \%$. As one can see, with Lorentzian components no additional powerlaw component is needed. One of the components (the one peaking around $1 \mathrm{~Hz}$ in the left panel of Figure 1.3 is narrower and can be identified as a quasi-periodic oscillation (QPO). The four Lorentzian components, from lower to higher characteristic frequencies, are called $L_{b}, L_{L F}, \mathrm{~L}_{\ell}$ and $L_{u}$, with a corresponding naming scheme for their characteristic frequency (Belloni et al. 2002). The full PDS is flat-topped at frequencies below $\nu_{b}$, declines as $\nu^{-1}$ (with bumps), then ends as $\nu^{-2}$ above $\nu_{u}$.

In the softer states, the characteristic frequencies increase and more obvious low-frequency QPOs appear. Three types of QPOs have been identified, each corresponding to one of the three branches in the ' $\mathrm{Z}$ ' path in the HID (see Figure 1.3). Correspondingly, they have been dubbed horizontal branch oscillations (HBO), normal branch oscillations (NBO) and flaring branch oscillations (FBO). NBOs are observed always around $6 \mathrm{~Hz}, \mathrm{FBOs}$ are in the range $\sim 4-20 \mathrm{~Hz}$, while HBOs span a larger range, from $\sim 1 \mathrm{~Hz}$ to dozens of hertz. (see van der Klis, 2006). HBOs are the most important for comparison with $\mathrm{BH}$ systems (although NBO/FBO also appear to have counterparts in BHBs): they are associated to a flat-top component which can be identified as a high-frequency extension of $L_{b}$. An example from XTE J1701-462 is shown in the right panel of Figure 1.3. The QPO often shows a second harmonic component and a peaked-noise component at around $\nu_{L F}$ (in grey in Figure 1.3).

High frequencies. At high frequencies $(\geq 100 \mathrm{~Hz})$ the picture is rather different. While for hard states only the high-frequency end of $L_{u}$ can be seen, the softer states are dominated by the so-called killohertz QPOs. Their main properties can be summarized in a few points:

- they are QPO peaks observed in the frequency range $200-1200 \mathrm{~Hz}$, with a $Q$ factor (defined as the centroid frequency divided by the FWHM, an indication of how coherent a peak is) that can be as low as a few, but in some case as high as 200 (see Barret et al., 2005ba). In 4U 1636-53, the 

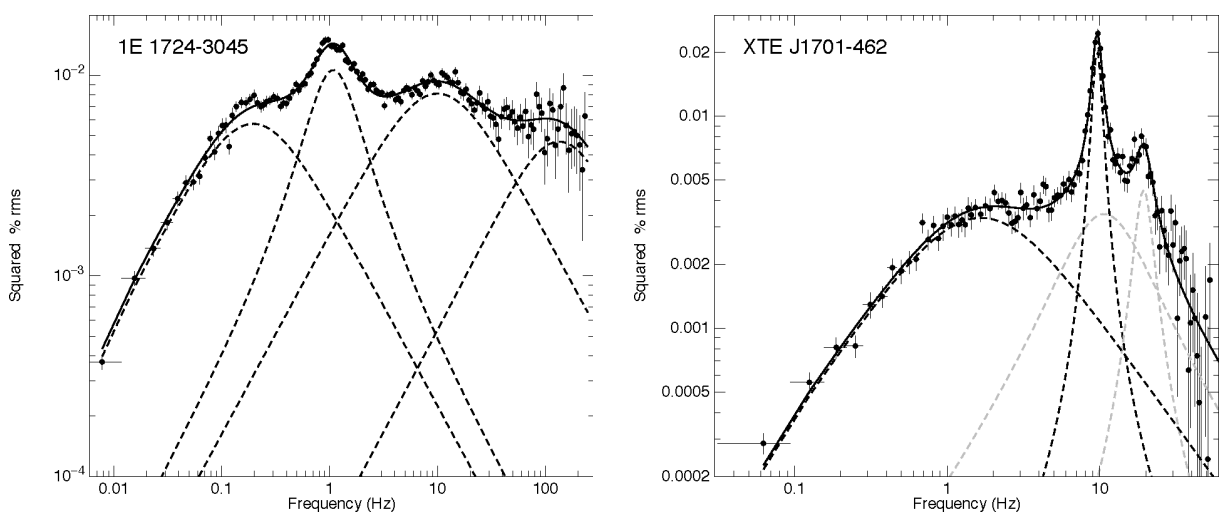

Figure 1.3 Examples of PDS (in $\nu P_{\nu}$ form) from NS LMXBs. Left panel: typical PDS from a hard state, from 1E 1724-3045. Right panel: PDS of XTE J1701-462 featuring a strong HBO. The grey lines indicate additional components (second harmonic and peaked noise, see text).

$Q$ factor of the lower $\mathrm{kHz}$ QPO has a maximum of $180 \mathrm{~Hz}$ at $\sim 850 \mathrm{~Hz}$, while the upper $\mathrm{kHz}$ QPO has always a $Q$ factor of a few (Barret et al. 2005a).

- there are two distinct peaks, often appearing together, referred to as the lower and the higher $\mathrm{kHz}$ QPO (see left panel in Figure 1.4). Their centroid frequencies vary on time-scales as short as minutes, moving roughly in parallel, i.e. maintaining (roughly) the same separation. The separation is for all systems around $\sim 300 \mathrm{~Hz}$ (with values between $200 \mathrm{~Hz}$ and $400 \mathrm{~Hz}$, see Méndez and Belloni, 2007). There is a good correlation also with spectral properties. In the right panel of Figure 1.4 all kHz QPO detections in the RXTE/PCA data of $4 \mathrm{U} 1636-53$ are shown in a plot of their centroid frequency versus hard colour. The two peaks are clearly separated into two different tracks, on which they move keeping roughly the same separation.

- their centroid frequency is generally correlated with source luminosity on short time-scales (hours), but the correlation disappears on long timescales and across different sources. In other words, while luminosity can span orders of magnitude, $\mathrm{kHz}$ QPOs remain in the $200-1200 \mathrm{~Hz}$ range. A possible interpretation of this phenomenon was presented by van der Klis (2001)

- there are no special frequencies associated to single sources (Belloni et al. 2007b). Their fractional RMS peaks at intermediate values and decreases at low and high centroid frequencies (Barret et al., 2005a). In the wellstudied case of $4 \mathrm{U} 1636-53$, the lower $\mathrm{kHz}$ QPO has a fractional RMS of 
$\sim 9 \%$ between 700 and $830 \mathrm{~Hz}$, to decrease above and below, while the upper $\mathrm{kHz}$ QPO has a fractional RMS peaking at $15 \%$ around $700 \mathrm{~Hz}$. The drop in fractional RMS and coherence has been interpreted as caused by the accretion flow reaching the innermost stable circular orbit (Barret et al. 2005a, 2006, 2007) but an alternative scenario was also suggested (Méndez, 2006).

While below $200 \mathrm{~Hz}$ there are low-frequency components that complicate the detection of a low-frequency $\mathrm{kHz} \mathrm{QPO}$, the sensitivity of powerspectral techniques to broad components does not depend on the centroid frequency. Therefore, there is no drop in sensitivity above $1200 \mathrm{~Hz}$, the maximum observed frequency (although the sensitivity decreases for broader peaks and at equal $Q$ factor a higher centroid corresponds to a broader peak).

- both lower and upper $\mathrm{kHz}$ show phase lags, but of different sign. A study of the properties of $\mathrm{kHz}$ QPOs in $4 \mathrm{U} 1608-52$ and $4 \mathrm{U} 1636-53$ showed that the lower $\mathrm{kHz}$ peak shows soft lags of the order of a few tens of microseconds, while the upper $\mathrm{kHz}$ peak shows hard lags of smaller amplitude (de Avellar et al., 2013).

- since their discovery, QPOs in NS LMXBs have been associated to the spin of the compact object. Now we know the spin period of the NS in a good number of systems and this can be tested. The results are puzzling. The first accreting millisecond pulsar that was discovered, SAX J1808.43658 with a spin frequency of $401 \mathrm{~Hz}$, showed two $\mathrm{kHz}$ QPO peaks at a separation of $\sim 195 \mathrm{~Hz}$, consistent with half the spin frequency (Wijnands et al., 2003). As new systems became available with two $\mathrm{kHz}$ QPO peaks and a spin period, their difference appeared to be close to $\nu_{\text {spin }}$ if $\nu_{\text {spin }}<400 \mathrm{~Hz}$, but close to $\nu_{\text {spin }} / 2$ if $\nu_{\text {spin }}>400 \mathrm{~Hz}$ (see Méndez and Belloni, 2007). However, $\Delta \nu$ is not constant for any single source, so the association cannot be exact. It was then shown that the existing data are also consistent with $\Delta \nu$ being independent of spin and distributed around $300 \mathrm{~Hz}$ in all sources (Méndez and Belloni, 2007, see also Figure1.19). However, examining sources separately one can see that the values of $\Delta \nu$ as a function of one of the two frequencies seem to 'know' the value of $\nu_{\text {spin }}$ or $\nu_{\text {spin }} / 2$ (see e.g. Barret et al., 2005a; Migliari et al., 2003).

\section{Frequency correlations}

Excluding NBOs and FBOs, the characteristic frequencies of all components in the PDS of NS LMXBs, whether in the form of QPO or broadband noise, correlate positively and different sources follow the same correlations (usually expressed as a function of the frequency of the upper $\mathrm{kHz} \mathrm{QPO}, \nu_{u}$ (see van Straaten et al., 2005; Linares et al., 2005). There 

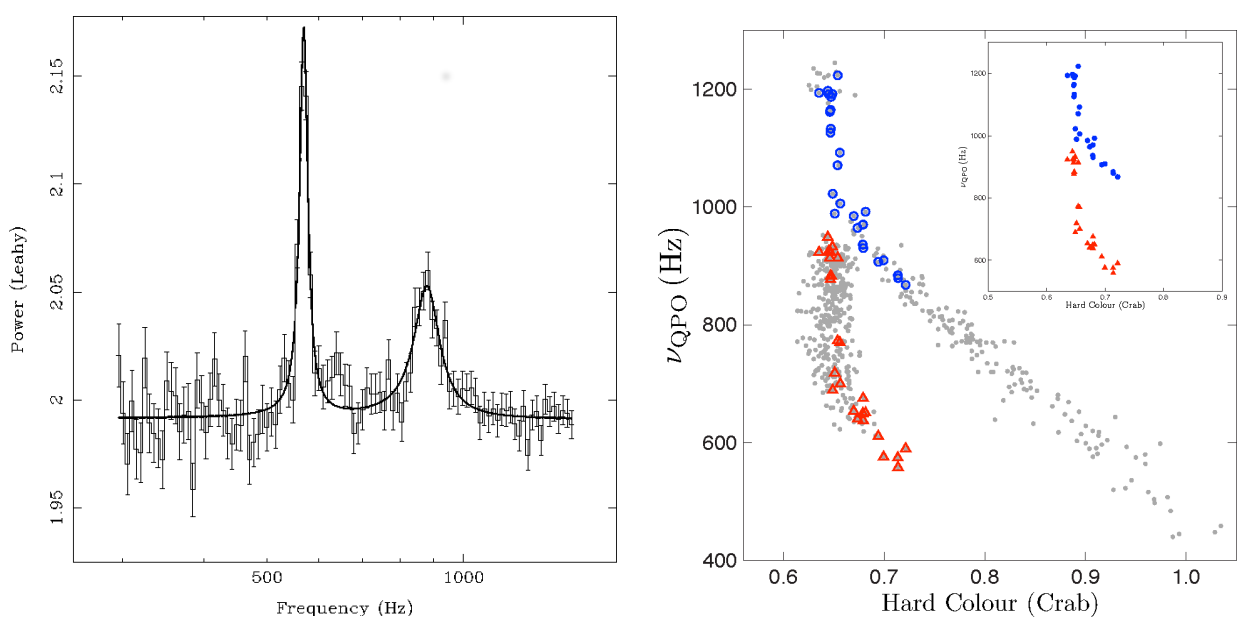

Figure 1.4 Left panel: PDS of the NS LMXB 4U 1608-52 where the 'twin peaks' of the $\mathrm{kHz}$ QPOs are evident (from Méndez et al., 1998). Right panel: $\mathrm{kHz}$ QPO centroid frequency as a function of X-ray colour for all $R X T E$ detections in $4 \mathrm{U} 1636-53$. Coloured dots correspond to pairs of simultaneously detected lower (red) and upper (blue) kHz QPOs. Black points indicate single detections. (From Sanna et al. 2012). (c)AAS. Reproduced with permission.

are two exceptions. The first is that the frequency of the so-called hectoHertz QPO component remains constant around $\sim 100 \mathrm{~Hz}$ as $\nu_{u}$ varies as much as one order of magnitude. The second is that for accreting millisecond X-ray pulsars, some systems show displaced correlations that can be reconciled with the others only by multiplying the frequencies of both $\mathrm{kHz}$ QPO by a factor $\sim 1.5$. Incidentally, with this factor the $\Delta \nu$ value for these sources would also become $\sim 300 \mathrm{~Hz}$ (Méndez and Belloni, 2007). Among these correlations, one is particularly important for theoretical models (see Section 1.5): that between $\nu_{H B O}$ and $\nu_{u}$. It can be fitted rather well with a quadratic dependence (Psaltis et al., 1999b), although in the case of GX 17+2 it was found to deviate for $\nu_{u}>1000 \mathrm{~Hz}$ (Homan et al., 2002).

While when examining $\mathrm{kHz} \mathrm{QPO}$ the data are usually plotted in terms of $\Delta \nu$ vs. $\nu_{u}$, a more direct plot is that of $\nu_{l}$ vs. $\nu_{u}$, i.e. the frequency of the lower QPO vs. that of the upper QPO (Belloni et al., 2007a). This plot for a set of sources can be seen in Figure1.5. One can see that multiplying both frequencies by a factor 1.5 (see above) would bring the two accreting millisecond pulsars SAX J1808.4-3658 and XTE J1807-294 on the general correlation. The lines in the figure will be discussed below. 


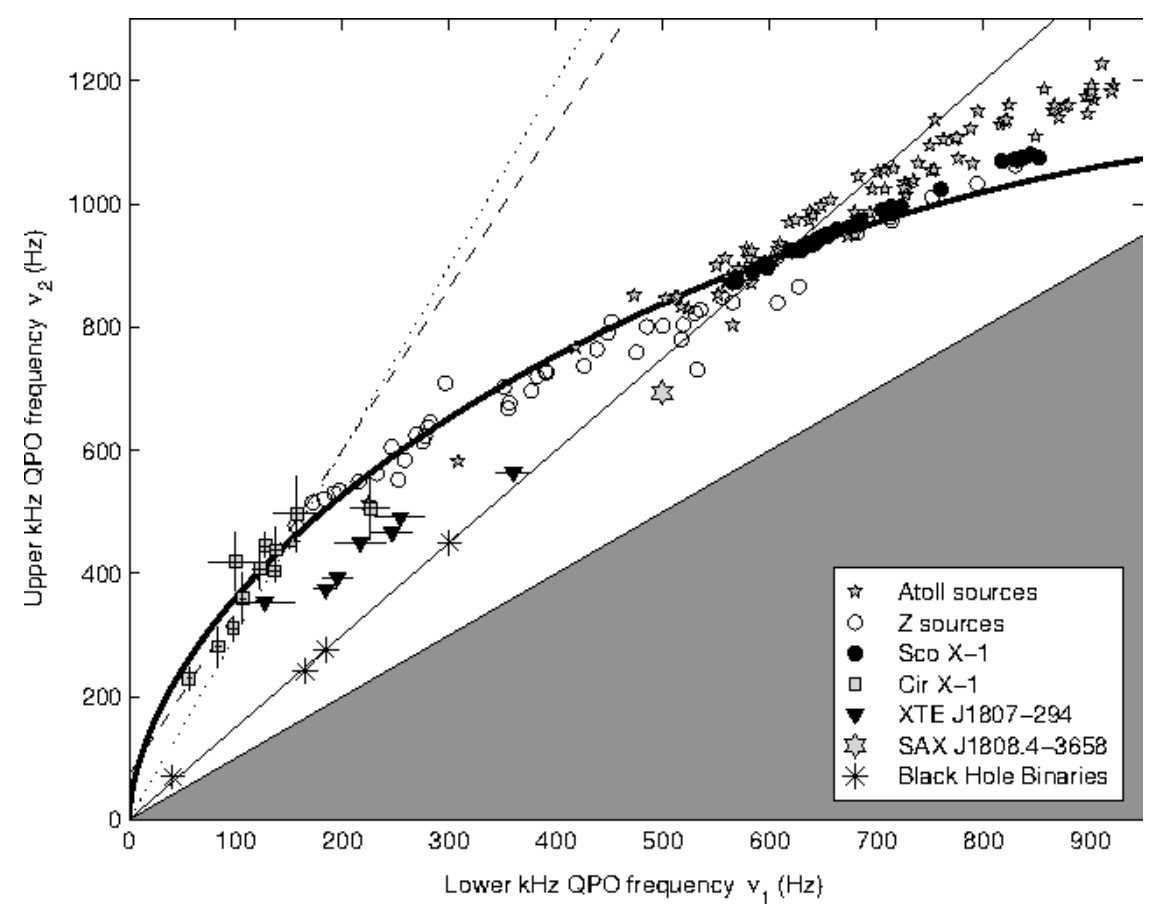

Figure 1.5 Correlation between lower and upper kHz QPO in NS LMXB. The points labeled 'Black Hole Binaries' are included for comparison, although some of them have since then been reconsidered (see text). The dark area correspond to lower frequency larger than upper frequency. The lines are: $\mathrm{y}=1.5 \mathrm{x}$ (solid thin), $\mathrm{y}=3 \mathrm{x}$ (dashed) and the relation between the periastron-precession and the Keplerian frequencies for a NS of $2 \mathrm{M}_{\odot}$ (solid thick). (From Belloni et al. 2007a).

\subsection{Black-Hole binaries}

\subsubsection{States and state-transitions}

Most of the known BHBs are LMXBs and most of them are transient. As in the case of NS systems, the large accretion rate swing in transients leads to a complex time evolution that can be subdivided into a number of separate states. These states are separated by marked transitions in either spectral or timing domain. Two main states were originally identified in the persistent system Cyg X-1 (Tananbaum et al., 1972) and more were added when the first transient BHBs were discovered and observed (Miyamoto et al., 1992, 1993, Belloni et al., 1996, 1997; Méndez and van der Klis, 1997). As shown below, a more solid state classification and outburst evolution has emerged, with obvious links to that of NS LMXB presented above.

Most known BHBs are transient, spending most of the time in their 'quies- 


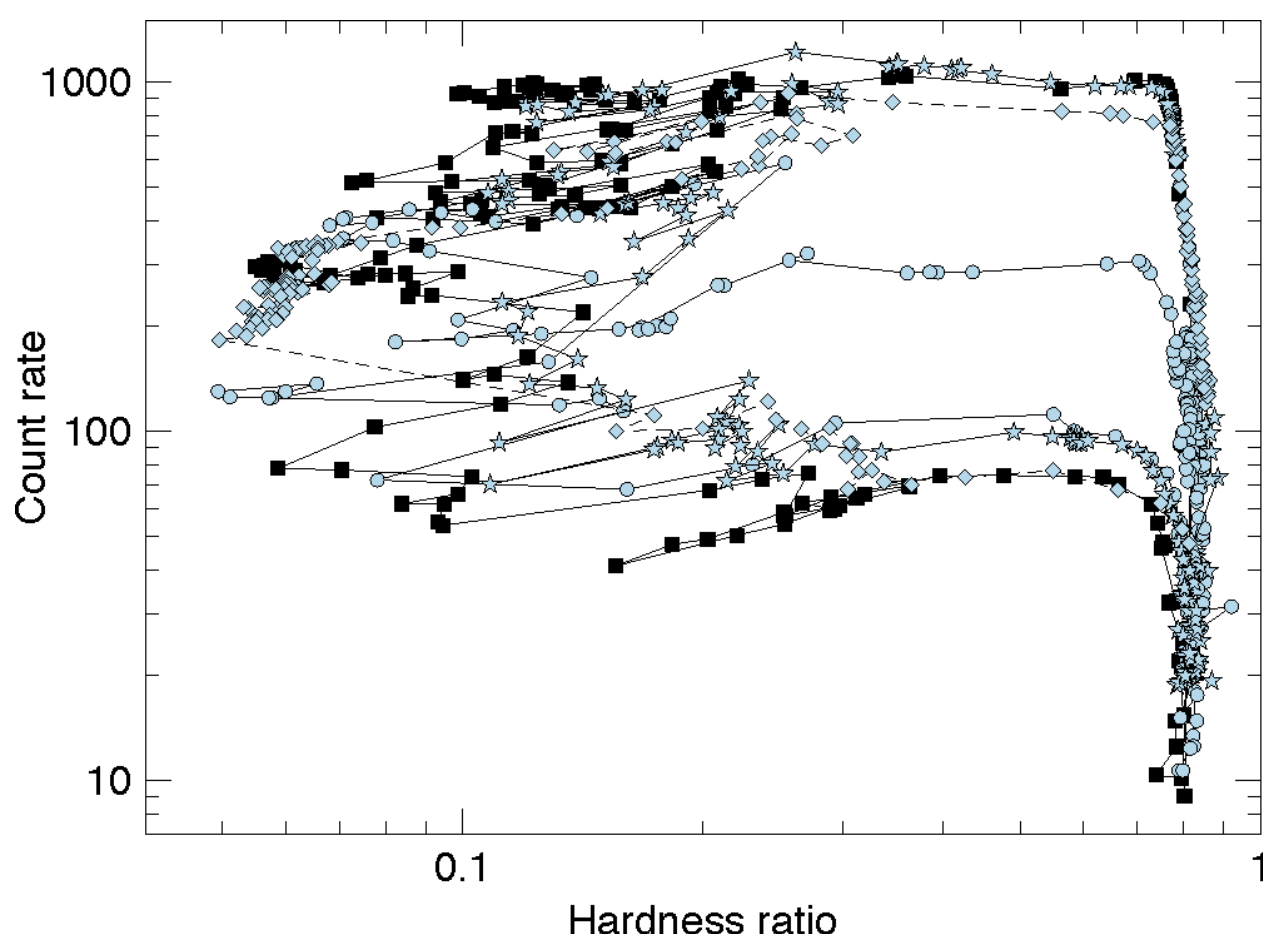

Figure 1.6 Hardness-Intensity diagram for four outbursts of GX 339-4 observed with RXTE. Each point corresponds to an observation and the lines connect them in time sequence. The dashed line represents a rather large time gap.

cent' state at a luminosity below $10^{33} \mathrm{erg} / \mathrm{s}$. At those low levels of accretion, $\mathrm{X}$-ray observations yield few photons and detailed studies are not possible (see Plotkin et al., 2015, and references therein). Recent observations confirmed that the energy spectrum in quiescence is softer than that in the hard state (see below). Transients leave the quiescent state with a recurrence time that ranges from months to decades, as the accretion rate onto the $\mathrm{BH}$ increases by several orders of magnitude due to the onset of thermal-viscous instabilities (see Lasota, 2016, for a review). During the outburst, the X-ray luminosity can approach the Eddington limit; typical durations are from a few days to several months, with a noticeable exception being the bright system GRS 1915+105, whose outburst started in 1992 and at the time of writing has not ended.

During an outburst, the spectral properties at X-ray energies change drastically, as does the fast variability: in addition to slow variations, rapid transitions are observed that led to the identification of source states, which 
are clearly related to the two original Cyg X-1 states. The sixteen years of dense observations with the RossiXTE satellite have led to a coherent picture to describe these variations in terms of few states, which have a clear correspondence to those of NS LMXBs shown above (whose outbursts are associated with the same instabilities). The X-ray light curves of different outbursts, even from the same recurrent source, can be very different, as they are influenced also by differences in the time evolution of the accretion rate. However, a clear pattern appears in most systems when a HID is produced. As in the case of NS LMXBs, different instruments will yield different HIDs and even different sources will be influenced by the interstellar absorption along their line of sight, but the pattern is strong and repeats in case of outbursts of the same source. The classical case is that of GX 339-4: Figure 1.6 shows the HID for four consecutive outbursts of this prototypical system. It is clear that the evolution of the four outbursts is the same, only the levels at which the main transitions take place and the time-scales for the overall evolution change. The first outburst, from 2002, is marked in black to make it more visible. Following it, as time progresses the source follows the q-shaped path in counterclockwise direction, starting from the bottom right. The full outbursts starts in quiescence, well below the diagram in the figure. As mentioned above, the quiescent energy spectrum is softer in quiescence, which means that below the figure frame the stem of the ' $q$ ' must bend to the left. The two original states correspond to the two vertical branches in the HID. The one at high hardness obviously corresponds to the low-hard state (LHS), the one on the left to the high-soft state (HSS). There are persistent systems that spend most of their time in the LHS, like Cyg X1 , and others that are found mostly in the HSS, like LMC X-3. Others, like LMC X-1, appear to be always in the same state, HSS in this case. The LHS branch is not really vertical, but shows a significant softening as the flux increases, while the large scatter observed in the HSS is partly caused by the magnification caused by the log scale. The similarity with the diagrams shown in Figures 1.1 and 1.2 is evident. The same hysteretic behaviour is visible, leading to a loop-like path traveled counterclockwise. This means that consistently the hard to soft transition takes place at a higher flux (and possibly accretion rate) than the reverse soft to hard transition. Cyg X-1 does show transitions between hard and soft, but within the uncertainties the two transitions appear not to show hysteresis (Belloni, 2010).

Particularly interesting are the points in between the LHS and HSS, which have been classified into two intermediate states. They are observed as transitions from hard to soft and from soft to hard, but also shorter excursions from the HSS into these states can be observed. The exact positions of the 
transitions cannot be seen in Figure 1.6, but can be measured precisely by monitoring other parameters such as the timing properties and infrared flux (see Belloni et al., 2005; Homan et al., 2005b). Two intermediate states have been identified, called hard-intermediate state (HIMS) and soft-intermediate state (SIMS) (see Belloni, 2010; Belloni et al., 2011; Belloni and Motta, 2016). The boundary between them is very well defined in terms of timing properties and transitions have been observed as fast as a few seconds. Although not too evident in Figure 1.6 because of the large scatter in the HS points (partly due to the log scale), there are several small transitions between intermediate states, which can be seen clearly in the fast timing properties (see below and Belloni et al., 2005; Casella et al., 2004). Not all sources display a perfectly shaped and repeatable ' $q$ ' diagram, but the general evolution is similar. Two systems, GRO J1655-40 and XTE J1550-564 have in addition a diagonal track in the upper part of the diagram, which has been called 'anomalous' or 'hyperluminous' state. Here the energy spectrum and timing properties are complex and it seems to correspond to cases when accretion rate continues to increase after the bright HSS is reached.

There is substantial agreement on the fact that it is mass accretion rate driving the outburst and therefore the evolution along the HID. On a 'clean' diagram like that in Figure 1.6 , the Y coordinate can be taken as a proxy for accretion rate: it increases from quiescence all the way to the upper left corner of the diagram, then decreases again down to quiescence. The vertical evolution is caused by accretion rate, but there is non agreement on what causes the horizontal evolution, in particular the two main transitions from hard to soft and from soft to hard. One possibility is that of a second parameter being involved, although a model based on the system memory has been proposed (Kylafis and Belloni, 2015). A systematic difference between the shape of HIDs of low- and high-inclination has also been found (Muñoz-Darias et al., 2013). Of course the interpretation of the evolution must rely ultimately upon detailed spectral analysis and the multiple components present in the energy spectra all contribute to the positioning in the HID; however, the HID is a clean way to present the evolution during an outburst and the inclination dependence would have been very difficult to discover from energy spectra alone (see Kylafis and Belloni, 2015; Petrucci et al. 2008).

In summary, a schematic HID for a $\mathrm{BH}$ transient is the one in Figure 1.7. where a second diagram is also included, the HRD, where the Y axis represents the integrated fractional variability in the Power Density Spectrum. It is evident that in this diagram there is no hysteresis and all points align along a general line from high variability in the LHS to very low levels of 


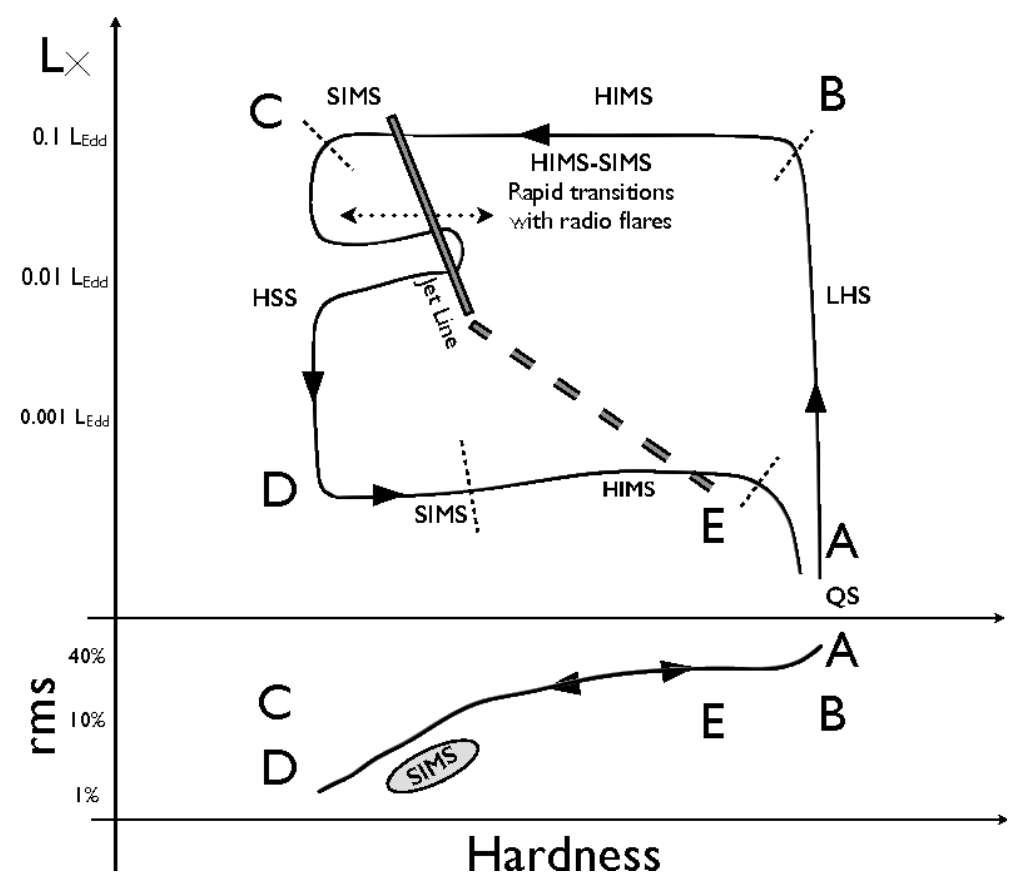

Figure 1.7 Schematic evolution of a BH transient in the HID (above) and HRD (below). The four states are marked. Large letters indicate corresponding points in the two diagrams. The 'jet line' marks the ejection of superluminal jets at relativistic speeds (From Belloni and Motta, 2016).

variability in the HSS. Only the points corresponding to the SIMS deviate from the general correlation, being characterised by lower variability (and very different timing features, see below)

\subsubsection{Energy spectra}

The Energy spectra of BHBs are also complex, but the absence of a solid surface simplifies their disentanglement. The differences between spectra in different states are major and the transitions difficult to follow.

- Low-Hard State The energy spectrum is, obviously, hard and its main component can be roughly approximated with a power law with a photon index 1.5-1.8 and a high-energy cutoff that can extend up to $\sim 100 \mathrm{keV}$ (see Figure 1.8). Even before the discovery of the cutoff, the presence of such a hard component was suggested as a direct evidence of a $\mathrm{BH}$ nature of the compact object, but a hard tail was then also observed in lowluminosity neutron-star binaries (see above). Most models associate this 
component to Comptonisation of softer photons from an optically thick accretion disk by electrons in a corona of either thermal or non-thermal nature (see Gilfanov, 2010), or possibly hybrid (see e.g. Del Santo et al., 2008). Alternative models for the hard emission have been presented: bulkmotion Comptonisation, where the disk photons are energised by inverse Compton interaction with a fast converging flow (see Turolla et al., 2002 , and references therein). Alternatively, since we know that relativistic collimated jets are present in hard states, the corona can be replaced with the base of the jet and the direct contribution of the jet to the observed emission has been considered (see Markoff, 2010). A common problem of Comptonisation models is that they depend on the geometry of the emitting region in a way that cannot be disentangled from intrinsic physical properties of the plasma, which means that additional information (such as fast timing variability or multi-wavelength observations) is needed in order to remove the degeneracy (see e.g. Petrucci, 2008). In addition, a strong components from reflection of hard photons off a thermal disk is observed, in the form of a broad emission line and a continuum component at higher energies (see Miller, 2007), as in the case of NS systems (see above).

For systems with low interstellar absorption, a thermal disk component is observed at low energies, modelled as a disk blackbody with a low temperature at the inner radius (see e.g. McClintock et al., 2003). However, there is disagreement as to what the inner radius of the disk is. Timing measurements suggest that the accretion disk is truncated at rather large radii, but the presence of strong reflection components leads to the opposite conclusion, namely that the disk extends to radii close to the $\mathrm{BH}$ (see Belloni and Motta, 2016, and references therein). Notice that the fits to the disk component are complicated by the presence of absorption and by the fact that at a fixed temperature the inner radius of the disk is determined by the normalization of the fit component (see Kolehmainen et al. 2014).

The LHS has been studied in detail in the persistent system Cyg X1, which spends most of its time in that state (Wilms et al., 2006) and in transients. As shown above, this state occurs at the beginning of the outburst, but also at the end, when the flux is lower, extending into the quiescent state. The ouburst decay properties also have been studied extensively (see Kalemci et al., 2013, Dinçer et al., 2014). The number of available observations at lower luminosity levels is much lower, due to the need of more sensitive instruments like those on board Chandra and $X M M-N e w t o n$. In the bright LHS, the spectrum hardens as the source 


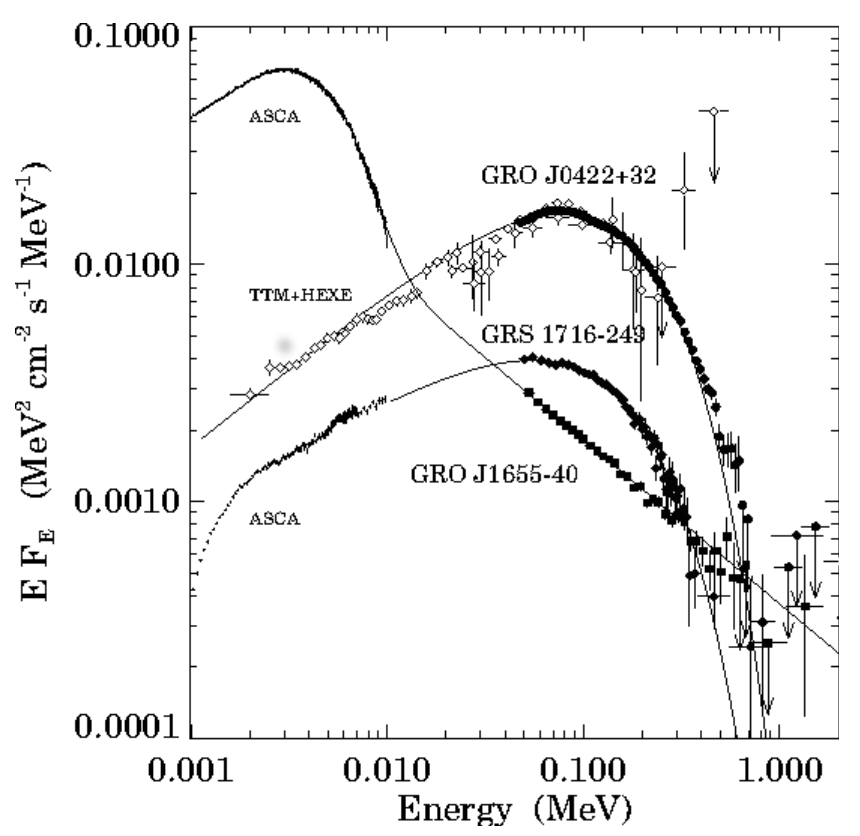

Figure 1.8 Examples of LHS (GRO J0422+32 and GRS 1716-249) and HSS (GRO J1655-40) broad-band energy spectra. (From Grove et al., 1998). (c)AAS. Reproduced with permission.

dims, but as luminosity goes down below $\sim 10^{-2} \mathrm{~L}_{\mathrm{Edd}}$ it starts softening until the spectral photon index reaches a 'plateau' value of $\sim 2$ (see Sobolewska et al., 2011, Plotkin et al., 2013).

- High-Soft State- In the HSS, the energy spectrum is dominated by a thermal disk component, with an inner temperature of the order of $1 \mathrm{keV}$ and extending to radii close to the BH. The original model of an optically thick and geometrically thin disc represents well the observations (Shakura and Sunyaev, 1973). However, the high statistics makes it possible to fit the spectrum with a more complex accretion disc model in order to take into account of all emission properties and estimate a precise value for the inner radius (see Davis et al., 2005). Assuming the radius is at the innermost stable circular orbit, this leads to the measurement of BH spins (see Middleton, 2016). As the HSS is observed over a wide range of luminosities and the inner radius is not observed to vary, the decay in mass accretion rate leads to a progressive softening of the spectrum. At high energies, an additional weak hard component is observed, often variable between observations (see Figure 1.8). The characterisation of this component is difficult due to its faintness and the dilution by the disk component at 


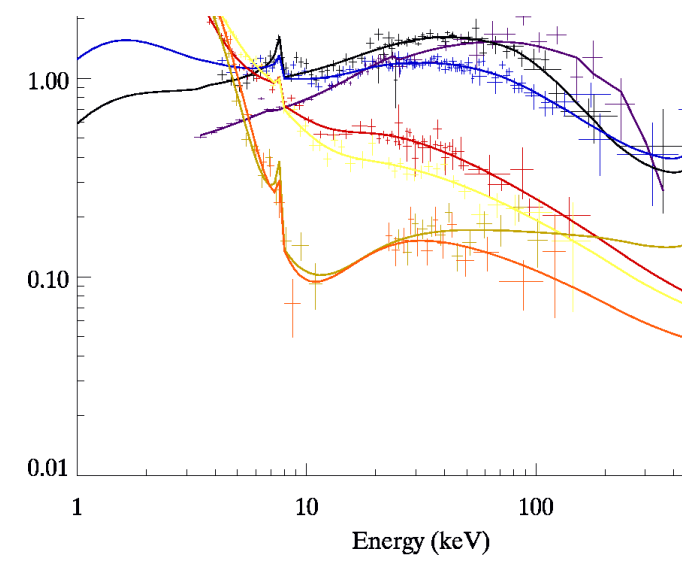

Figure 1.9 Examples of intermediate-state energy spectra from GX 339-4. The violet spectrum is an example of LHS for comparison. The spectral fits are with a hybrid Comptonisation model, a thermal disk and a reflection complex. (From Del Santo et al., 2008)

low energies (Grove et al., 1998, Zdziarski et al., 2012). Reflection features have been observed also in the HSS, where again measurements are complicated by the direct disk emission (Steiner et al., 2016).

- Intermediate States The energy spectrum during intermediate states is indeed intermediate. The transition from a hard spectrum dominated by a Comptonisation component featuring a weak disk component to a soft spectrum dominated by a disk component is gradual (see Figure 1.9). Fits with hybrid Comptonisation models indicate that the spectral evolution is drive by a decrease in the ratio between electron heating and soft cooling flux from the disk (Del Santo et al., 2008). In GX 339-4, the high-energy cutoff in the spectrum, which decreased from $120 \mathrm{keV}$ to $60 \mathrm{keV}$ as the flux increased in the LHS, showed a marked increase in the HIMS up to above $100 \mathrm{keV}$, after which it became too high to be detected by RXTE (Motta et al. 2009). Whether it was still detected in the only SIMS observation is unclear, as only one observation was made in that state. The same behaviour was observed in GRO J1655-40 (Motta et al., 2009; Joinet et al., 2008). On the return track from soft to hard state, sources go through the same intermediate states (see Figures 1.6 and 1.7 ). The main spectral differences compared to the high-flux branch are a softer disk component and a flatter hard component, whose photon index reaches LHS values at the start of the HIMS (Stiele et al., 2011). 


\subsubsection{Timing properties}

As mentioned above, the movement on the HID is determined by spectral variations, but the discrete states are defined in terms of timing properties, which show marked variations corresponding to specific hardness levels. In analogy of what done above for NS systems, I will present the main characteristics of timing features distinguishing low and high frequencies (see Motta, 2016, for a recent review).

\section{Low frequencies}

In addition to noise components that can be very strong and completely dominate the variability, three LFQPOs have been identified, corresponding to different source states (see Casella et al., 2005). Their properties are different and they have been detected simultaneously in a few cases, confirming that they are different signals, although of course this does not rule out the same physical origin.

- Type-C QPO. These are the most common low-frequency oscillations, observed in the HIMS and in the bright LHS. They have been detected also in the HSS (see e.g. Motta et al., 2012). Its centroid frequency is anti-correlated with hardness: in the LHS it can be below $0.1 \mathrm{~Hz}$, in the HIMS it is typically $1-10 \mathrm{~Hz}$ and in the HSS it can reach $30 \mathrm{~Hz}$ (see Belloni, 2010, Belloni and Motta, 2016). Type-C QPOs are rather strong, reaching up to $20 \%$ fractional RMS, and narrow, with a $\mathrm{Q}$ factor $\sim 10$. Their centroid frequency varies over a large range $(0.1-15 \mathrm{~Hz})$ and they appear associated to band-limited noise components (see Figure 1.10). Often a second harmonic peak is detected, rarely a third harmonic. In many cases, a sub-harmonic feature at half the QPO frequency is also seen (see e.g. Takizawa et al., 1997; Casella et al., 2004; Rao et al., 2010; Motta, 2016). The frequency of type-C QPO correlates positively with the characteristic frequencies of noise components (see below). A strong anti-correlation is observed between the QPO centroid frequency and the fractional RMS measured over a broad frequency range (not the RMS of the QPO itself, Casella et al., 2005; Motta et al., 2011).

- Type-B QPO. These oscillations are the defining trait of the SIMS. The peaks are relatively strong around $\sim 4-5 \%$ RMS and with $Q$ larger than 5 . In contrast with type $\mathrm{C}$, they are observed over a limited range of frequencies $(\sim 1-7 \mathrm{~Hz})$. They are sometimes observed with a second harmonic and a sub-harmonic, which at times can be as strong as the fundamental (the so-called 'cathedral' QPO, see Casella et al. 2004, and Figure 1.10, left panel). They are associated with a power-law noise with 

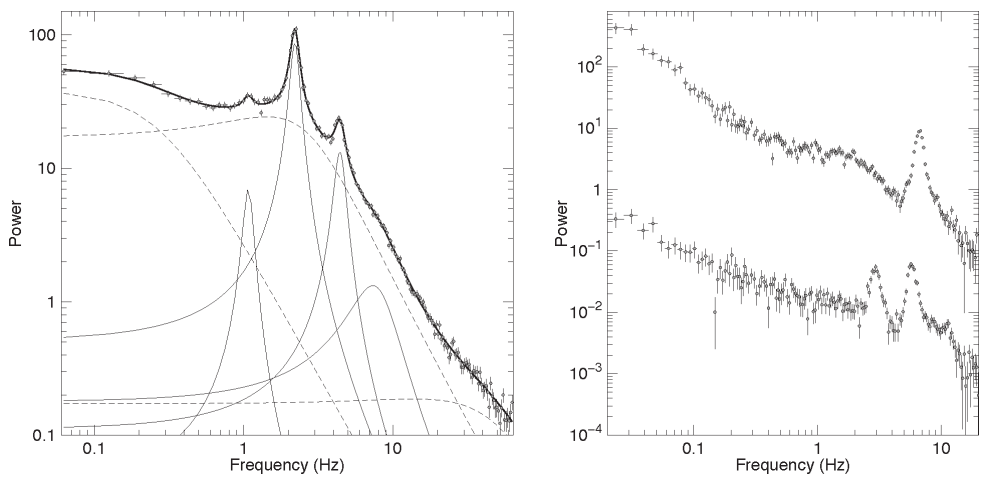

Figure 1.10 Left panel: PDS from GRS 1915+105 in its C state, corresponding to the HIMS (from Ratti et al., 2012). Right panel: two examples of type-B QPO from GX 339-4 in the SIMS, shifted in power for clarity. In the top one the QPO does not have harmonics, in the second one the subharmonic peak is as strong as the fundamental (the so-called 'cathedral' $\mathrm{QPO})$.

a few \% fractional RMS, which means the QPO dominates the observed variability (see Figure 1.10). The centroid frequency is observed to vary rapidly with a typical time-scale of $\sim 10$ s. (Nespoli et al., 2003, Casella et al., 2004; Motta et al., 2011). Rapid transitions have been observed between this QPO and the other types, with transition times as short as a few seconds (Nespoli et al., 2003, Casella et al., 2004; Motta et al., 2011). In GX 339-4, a strong correlation was found between centroid frequency and source flux above $6 \mathrm{keV}$, where the disk contribution is negligible. For type-C QPOs there is a general anti-correlation, although not so tight (Motta et al., 2011). A few observations at very high flux of GX 339-4 have led to the simultaneous detection of a type-B and a type-C QPO, confirming that the two oscillations are different (Motta et al., 2012).

- Type-A QPO. This QPO is also associated with weak power-law noise, but the peak is much more blunt, with $\mathrm{Q} \leq 3$. Its centroid frequency is always around $6-8 \mathrm{~Hz}$. Given these characteristics that make it more difficult to detect and characterise, not many details are know. No harmonic content appears to be present, although the low coherence of the peak make it difficult to study additional peaks. These QPOs are observed in the HSS, close to transitions from the SIMS (see Casella et al., 2004; Motta et al. 2012).

- Noise components

In the LHS, the variability is very strong and dominated by band-limited noise components. As shown in the HRD in Figure 1.7, the total fractional 


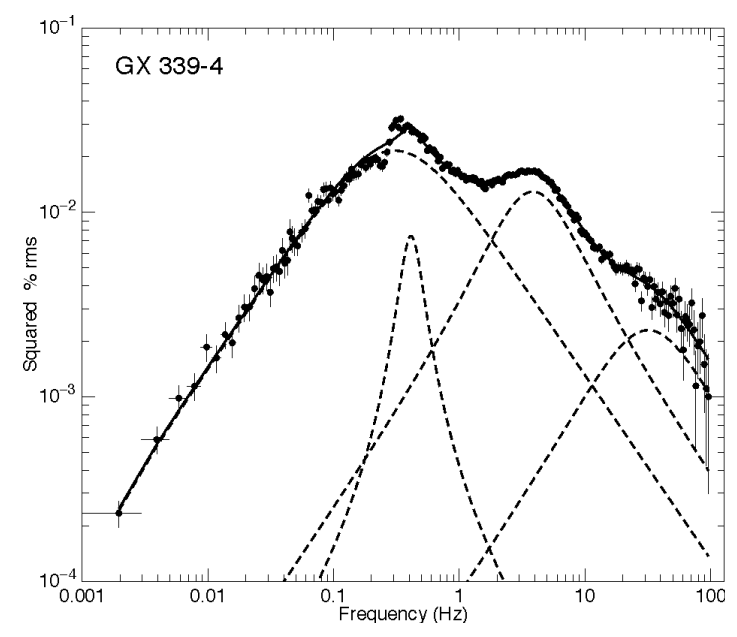

Figure 1.11 Power density spectrum of a set of RXTE observations of GX 339-4 in hard state. The best fit with four Lorentzian components is shown. The quality of the fit in the $0.1-1 \mathrm{~Hz}$ range is poor because of variability between the observations (see Nowak, 2000).

RMS can be as high as $40 \%$. As in the case of NS LMXBs presented above, the PDS can be decomposed into the sum of at least four separate components, called called $L_{b}, L_{L F}, \mathrm{~L}_{\ell}$ and $L_{u}$ (see Belloni et al., 2002, and Figure 1.11, left panel). The first fits with flat-top Lorentzian components were made by Olive et al. (1998) on a NS and a BH system, but the first systematic attempt of replacing power laws with broad Lorentzians was presented by Nowak (2000) and further generalized by Belloni et al. (2002). The $L_{b}, \mathrm{~L}_{\ell}$ and $L_{u}$ are broad, usually represented by zero-centered Lorentzians, while $L_{L F}$ is the type-C LFQPO more evident in the HIMS. A study of a large number of $R X T E$ observations of CygX-1, most of which taken in the LHS, revealed a complex relationship between the parameters of the four Lorentzians in this source (Pottschmidt et al. 2003$)$. In X-ray transients, as flux increases and the spectrum softens in the LHS, the characteristic frequencies of the four components increase and their fractional RMS decreases, until at the onset of the HIMS $\mathrm{L}_{\ell}$ and $L_{u}$ are normally not detected anymore due to their frequency being too high and their RMS too low. Since these results come from the analysis of $R X T E$ data, the $R X T E / \mathrm{PCA}$ instrument has a lower energy threshold of $\sim 3 \mathrm{keV}$ and most X-ray transients suffer of high levels of interstellar absorption, in most cases the PCA only detects photons from the high-energy component and cannot see the thermal disk component (see above). Therefore, the 
observed variability can only originate from the Comptonised component. However, observations of GX 339-4 with XMM-Newton have led to the discovery that in the LHS on time-scales longer than a second the variability of the thermal disk, detected below $3 \mathrm{keV}$, leads that of the hard component, while the reverse happens on faster time-scales. The conclusion is that in the LHS the thermal disk variability is responsible for the hardcomponent one, while on short time-scales disk heating effects reverse the casual connection (Uttley et al., 2011). This result is supported by the observation that on long time-scales the thermal-disk variability is stronger than the one from the Comptonised component (Wilkinson and Uttley, 2009). Another important observational results, which appears to hold in other states other than the LHS, is the so-called 'RMS-flux' relation: the total (not fractional) RMS is linearly correlated with the observed count rate. This property cannot be explained in terms of older models involving the superposition of randomly occurring 'shots' and is naturally interpreted in terms of propagating-fluctuation models (see Lyubarskii, 1997), in which the slower variations from the outer parts of the accretion disk propagate inwards and are then further modulated at higher frequencies by more internal portions of the disk.

In the HIMS the noise is dominated by the high-frequency extension of $L_{b}$ and the characteristic frequencies continue to increase as the energy spectrum softens. A thermal disk component starts contributing to the RXTE energy band, but the fractional RMS at those energies decreases in a way compatible with all variability being associated to the hard component, while the detected thermal-disk flux is not variable. This is in marked contrast with what discussed above for the LHS, meaning that the disk varies more when not observable, but becomes 'quiet' when dominating the flux. Broader studies have shown that the RMS-flux relation originally observed for the LHS is present across states, with rather complex properties (see Muñoz-Darias et al., 2011; Heil et al., 2012).

After the transition to the SIMS the band-limited noise components disappear and are replaced by a steep power-law component. The variability can be as low as $\sim 1$ fractional RMS.

\section{High Frequencies}

The first High-frequency QPO (HFQPO) from a BHB was detected during the first year of RXTE operation in the bright and peculiar transient GRS 1915+105 (Morgan et al., 1997). Its frequency was around 65-67 Hz, with a $Q$ value of $\sim 15$ and a fractional RMS amplitude of $1-1.6 \%$. However, after sixteen years of observations of BHBs, only few detections in 

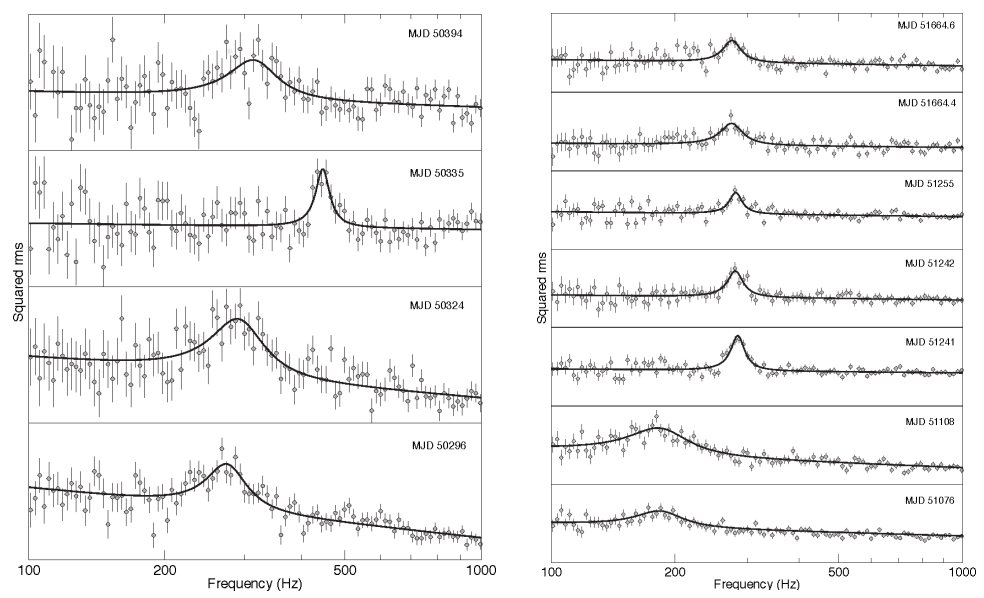

Figure 1.12 Left panel: detections of single HFQPO peaks in GRO J165540 , peaking at $\sim 300 \mathrm{~Hz}$ and $\sim 450 \mathrm{~Hz}$. Right panel: detections of single HFQPO peaks in XTE J1550-564, peaking at $\sim 180 \mathrm{~Hz}$ and $\sim 280 \mathrm{~Hz}$ (from Belloni et al. 2012).

other systems have been found (see Belloni et al., 2012; Motta, 2016). These are XTE J1550-564 (Remillard et al., 1999b; Homan et al., 2001; Miller et al., 2001; Remillard et al., 2002), GRO J1655-40 (Remillard et al., 1999a: Strohmayera, 2001), XTE J1650-500 (Homan et al., 2003), H1743-322 (Homan et al., 2005a: Remillard et al., 2006c), and IGR J17091-3624 (Altamirano and Belloni, 2012). Detections were reported also for XTE J1859+226 (Klein-Wolt et al., 2004) and 4U 1630-47 (Klein-Wolt et al., 2004) although their significance appears to be low.

From this limited number of detections we have now the following observational picture, where GRS $1915+105$ is dealt with as a separate case.

- In one source, GRO J1655-40, there is a significant detection of two simultaneous peaks, at $\sim 300 \mathrm{~Hz}$ and $\sim 440 \mathrm{~Hz}$, with other detections of single peaks around the same value (see Fig 1.12 , left panel), although statistically different (Strohmayera, 2001; Belloni et al., 2012; Motta et al. 2014a). In XTE J1550-564 two separate peaks have been detected around $180 \mathrm{~Hz}$ and $280 \mathrm{~Hz}$ (see 1.12 , right panel), but not at the same time, although in one case there is a hint of a second peak (Miller et al., 2001). It is tempting to consider them two separate peaks, although comparisons based on phase lags suggest that they might be the same component changing frequency (Méndez et al., 2013). Besides this consideration, the variations in the centroid frequency of all HFQPOs are minor, suggesting they are associated to fixed frequencies. 

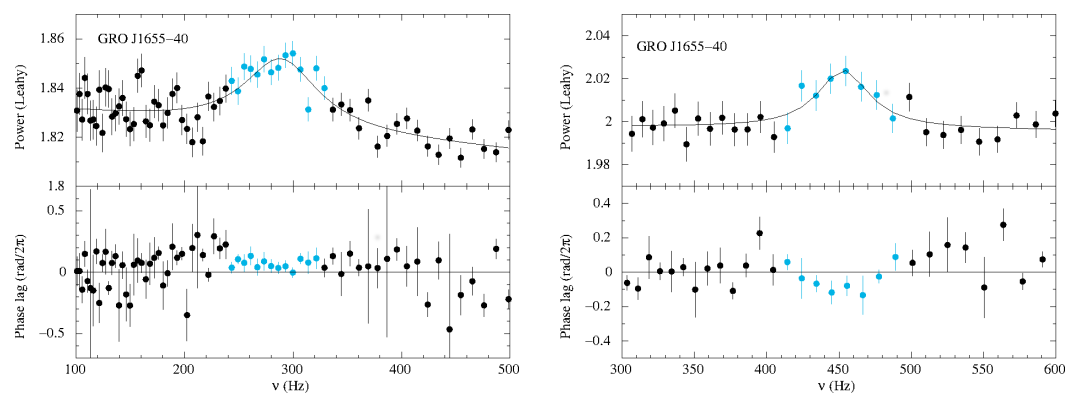

Figure 1.13 Top panels: detections of single HFQPO peaks in GRO J165540 , peaking at $\sim 300 \mathrm{~Hz}$ and $\sim 450 \mathrm{~Hz}$. Bottom panels: corresponding phaselag spectra. (from Méndez et al. 2013).

- The fractional RMS of HFQPOs is between 1 and 6 , but it is strongly energy dependent, being much stronger at high energies. Quality factors are in the range $1-5$.

- All detections correspond to observations at high flux. While this could be due to a sensitivity bias for weak signals, in GRO J1655-40 and XTE J1550564 it is clear that all detections correspond to the 'anomalous' or 'hyperluminous' state, which can be observed at higher fluxes than those shown in the diagrams above (see Belloni, 2010; Belloni et al., 2012). No detection is reported in the LHS and in the HSS, indicating that they are associated to intermediate states, while connected to the hard component as their RMS is highest at energies $>10 \mathrm{keV}$.

- When two peaks are observed simultaneously, in GRO J1655-40 and GRS 1915+105, the lower peaks has soft lags and the upper peak has hard lags (see Figure 1.13, Méndez et al., 2013). For XTE J1550-564, as mentioned, the $180 \mathrm{~Hz}$ and $280 \mathrm{~Hz}$ peaks have similar lags, which suggests they might be the same peak at different frequency.

- In the case of GRO J1655-40 and XTE J1550-564, the frequencies of the two peaks are around a 3:2 ratio (see Abramowicz and Kluźniak, 2001).

- The case of GRS 1915+105, as in many other respects, needs to be treated separately. The original detection was of a peak at $65-67 \mathrm{~Hz}$ (Morgan et al., 1997). Since the observations where the oscillation was found showed very strong variability on time-scales of $10-20 \mathrm{~s}$, a flux-selected analysis led to the discovery of another peak at $27 \mathrm{~Hz}$, not simultaneous to the $67 \mathrm{~Hz}$ one (Belloni et al. 2001). Shortly afterwards, a $41 \mathrm{~Hz}$ peak simultaneous to the $67 \mathrm{~Hz}$ one was discovered from a subset of $R X T E$ observations (Strohmayerb, 2001). A semi-automatic study of all RXTE observations for a total exposure time of $5 \mathrm{Ms}$ led to the detection of $51 \mathrm{QPO}$ peaks 

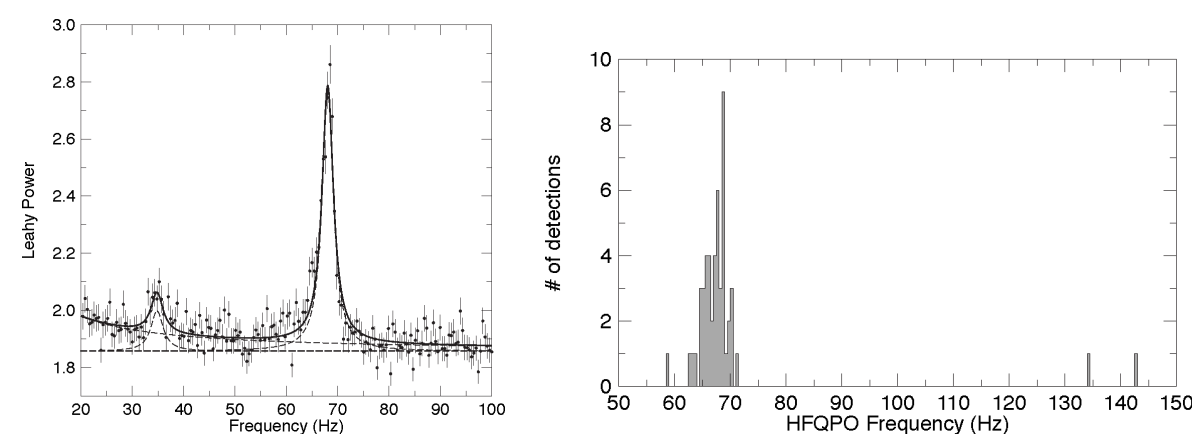

Figure 1.14 Left panel: double HFQPO in GRS 1915+105 (from Belloni and Altamirano, 2013a). Right panel: distribution of the detection of single HFQPOs in GRS 1915+105 (from Belloni and Altamirano, 2013b).

in as many observations. Most of the centroid frequencies were in the $63-71 \mathrm{~Hz}$ range (see Figure 1.14 , left panel), indicating that $\sim 67 \mathrm{~Hz}$ (the average value) must be a fundamental frequency in the system (Belloni and Altamirano, 2013b). A time-resolved analysis indicates that the detections are confined to a restricted region of the HID of GRS 1915+105 (which has a peculiar and complex shape), again corresponding to the highest count rates (Belloni and Altamirano, 2013b). An additional peak at $34 \mathrm{~Hz}$ (see 1.14 , right panel), simultaneous with the $67 \mathrm{~Hz}$ one and consistent with half of its frequency, was found in data corresponding to a subregion in the HID, just as the $41 \mathrm{~Hz}$ one corresponded to a separate subregion (Belloni and Altamirano, 2013a). This system showed therefore multiple centroid frequencies: $67 \mathrm{~Hz}, 27 \mathrm{~Hz}$, a pair $41-67 \mathrm{~Hz}$ and a pair 34-67 Hz. The sequence 27:41:67 corresponds to 2:3:5, while 34:67 is 1:2. Independent of the model, $67 \mathrm{~Hz}$ is too low to be the Keplerian frequency at the innermost stable circular orbit for GRS 1915+105, being too slow even for the highest mass allowed for the $\mathrm{BH}$ in the system (Reid et al., 2014) and zero spin.

\subsection{Comparison between NS and BH systems}

\section{Comparison}

When a new galactic transient X-ray source is observed in the sky, even in absence of detected pulsations or X-ray bursts, unambiguous telltales of the presence of a NS, in most cases the X-ray properties are sufficient for the identification of the nature of the compact object, although not a conclusive one as no definitive criterion for the presence of a $\mathrm{BH}$ in the system has 
yet been found. Below, I will outline the main differences and similarities in $\mathrm{X}$-ray properties between classes of sources.

\subsubsection{Quiescent emission}

$\mathrm{BH}$ transients in quiescence have a very low X-ray emission that contrasts with the relative optical brightness of the accretion disk, a discrepancy that can be solved with the presence of an advection-dominated accretion flow (ADAF), where the flow is radiatively inefficient (see Narayan et al., 1996). However, in the case of a NS, the advection energy stored in heat in the ADAF is not lost through the event horizon and must be released on the star's surface. Therefore, the quiescent luminosity of a NS transient is expected to be lower than in the $\mathrm{BH}$ case, given the same accretion rate. Accretion rate depends on the binary orbit, with tighter systems being fainter (see Menou et al., 1999), but is not expected to be different between different classes of systems. Deep observations with current instruments confirm both statements (see Figure 1.15). The theoretical situation is however more complicated and the claim that this results is an indication of the existence of event horizons has been critically discussed by Abramowicz et al. (2002).

\subsubsection{X-ray bursts}

Thermonuclear X-ray bursts are X-emission originating on the surface of a NS and as such a direct evidence of the presence of said surface. It follows that, by definition, none of the currently known BHBs has shown an X-ray burst. However, it has been suggested that the absence of X-ray bursts can be taken as an indication of the absence of a surface and therefore as an indication of the BH nature of a source (Narayan and Heyl, 2002). A statistical analysis of RXTE data in relation to burst models confirmed numerically this statement (Remillard et al., 2006b). Also in this case, Abramowicz et al. (2002) have criticised this approach as a detection of the presence of an event horizon.

\subsubsection{Energy spectra}

As mentioned in the introduction, several spectral 'signatures' have been proposed in the past to identify the presence of a $\mathrm{BH}$ in an X-ray binary The very soft thermal component observed from the accretion disk in soft states cannot be considered as a signature, as low-B neutron-star binaries can have accretion disks extending to roughly the same inner radius as BHs. 


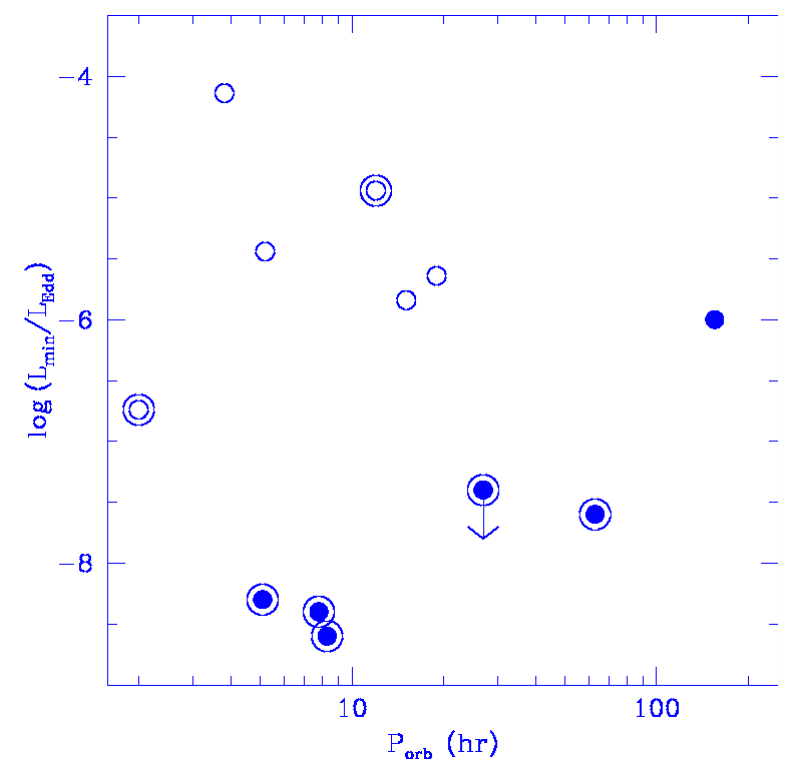

Figure 1.15 Quiescent luminosity for X-ray transients as a function of orbital period. Only the lowest observed value for each system is shown. Filled symbols represent BH binaries, empty symbols neutron-star binaries. (From Garcia et al., 2001). (c)AAS. Reproduced with permission.

Measurements of inner disk radii through high-resolution spectroscopy are also unlikely to yield results that would allow a strong identification. A good discussion of this comparison throughout the full spectrum can be found in Lin et al. (2007), but no clear dividing line between classes of systems is found.

The hard component (often referred to as 'hard tail'), which in the past was compared simply in terms of flux or power-law index, can now be also analysed in terms of more complex physical models. Recently, Wijnands et al. (2015) reported the results of the spectral analysis of a sample of NS LMXBs showing that at lower luminosities, in the range $10^{34}--10^{35} \mathrm{erg} / \mathrm{s}$, $\mathrm{BH}$ systems have systematically harder (lower power-law photon index) spectra than NS (see 1.16). The separation between classes of sources appears to be marked and additional data will be able to test this method. Another recent proposal for a clear distinction between classes of sources was presented by Burke et al. (2016). Fits with Comptonisation models to RXTE data of systems in the hard state show that BH and NS systems display different values of parameters. BHs correspond to higher values of the Comptonisation parameter $y$ and higher amplification ratio $A$ (hard flux 


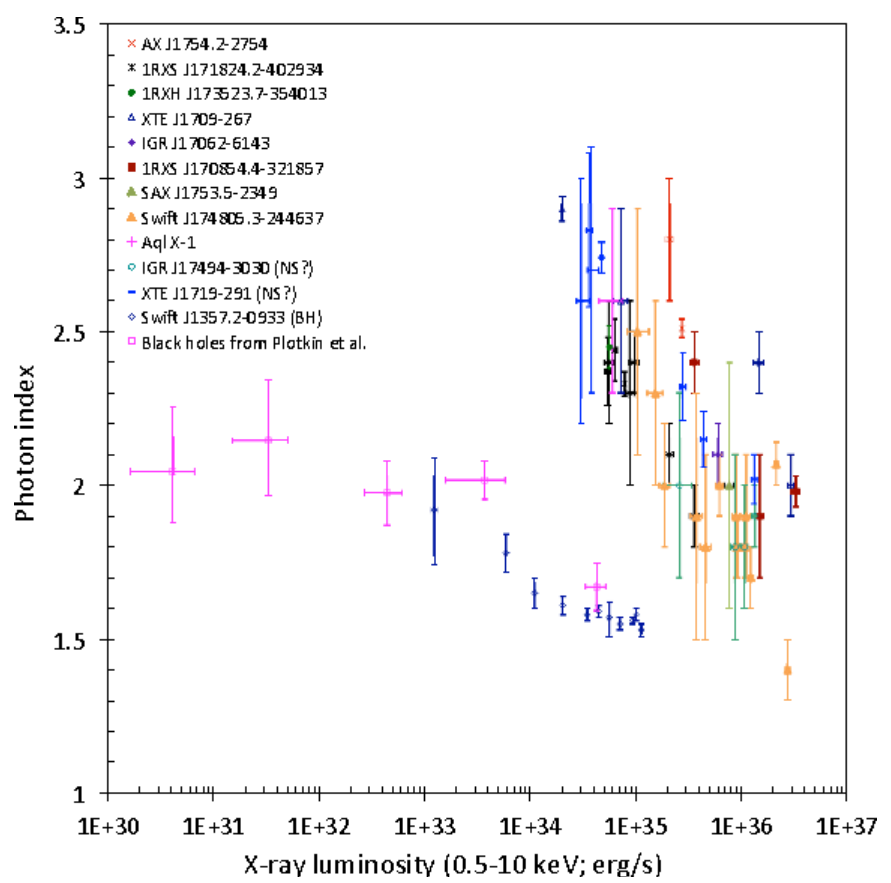

Figure 1.16 Plot of photon index - luminosity for a sample of NS and BH X-ray binaries (from Wijnands et al. 2015). (c)AAS. Reproduced with permission.

divided by seed flux). While the $y-A$ relation appears to be common, there is a clear segregation (Burke et al., 2016).

Comparing the evolution of both transient and persistent systems in the HID, one can see that there is no major difference, indicating that the underlying physics must be, as expected, the same (see Muñoz-Darias et al., 2014). At the same time, results like those of Burke et al. (2016) and Lin et al. (2007) indicate that the same models can be applied, with significant differences in physical parameters.

\subsubsection{Time variability}

The comparison of fast-timing properties in BH and NS sources is complex, but very promising. The deconvolution of PDS into a combination of Lorentzian components (Olive et al., 1998; Belloni et al., 2002) has allowed a homogeneous treatment of all systems, allowing to link different classes of sources across different states (see Figures 1.3 and 1.11). Comparing the two figures one can see that in the hard states the power distribution appears 
similar: a more peaked (QPO) component, a broad component at lower frequencies $\left(L_{b}\right)$ and two broad components at higher frequencies $\left(L_{\ell}\right.$ and $\left.L_{u}\right)$. The main difference between the NS and $\mathrm{BH}$ case is that the relative power of $L_{\ell}$ and in particular $L_{u}$ are higher in the NS case. Indeed, the difference in power above $10-50 \mathrm{~Hz}$ has been proposed as an empirical method to distinguish BH from NS binaries (Sunyaev and Revnivtsev, 2000). In the hard states, no counter-example has been found. Sunyaev and Revnivtsev (2000) interpret it as an effect of the presence of a boundary layer in NS binaries, although the presence of the same number of components in the PDS seems not to be consistent with this idea.

The identification of similar components in both classes of systems (see Belloni et al., 2002) has allowed a detailed comparison. Identifying the most common QPO in BHBs (type-C) with the HBO in NS systems (see below) and comparing its frequency with that of the $L_{b}$ component has led to a rather tight correlation (called WK correlation, see Figure 1.17 and Wijnands and van der Klis, 1999). Z sources appear to deviate from the correlation, which otherwise extends over three orders of magnitude (see $\mathrm{Bu}$ et al., 2015, for an updated version). Belloni et al. (2002) added data to the correlation and noticed that there is a parallel correlation following a 1:1 relation, when characteristic frequencies are considered. However, the noise component related to these points was found to be an additional one $\left(L_{h}\right)$. The WK correlation connects two homogeneous observables through different classes of systems and indicates that the underlying phenomenon does not depend on the nature of the compact object. There is the indication of a segregation of BHB points to lower frequencies, but this is expected on the basis of mass scaling.

There is another correlation that links different classes of systems over three orders of magnitude, the so-called PBK correlation (Psaltis et al. 1999a: Belloni et al., 2002). Unlike the WK correlation, here non-homogeneous quantities are included. The version of the correlation by Belloni et al. (2002) is shown in Figure 1.18, modified to remove incorrect points and to add the two points from Motta et al. (2014a b) (see below). For NS binaries with $\mathrm{kHz}$ QPO, what is plotted in Figure 1.18 is the frequency of the HBO versus that of the lower $\mathrm{kHz}$ QPO. For hard state sources, both containing $\mathrm{BH}$ and NS, the plotted quantities are the type- $\mathrm{C} \mathrm{QPO}$ for $\mathrm{BH}$ or the low-frequency $\mathrm{QPO}$ for NS versus the characteristic frequency of the lower of the high-frequency broad component $L_{\ell}$. Added to the plot are the two only $R X T E$ detections of a HFQPO and a simultaneous type-C QPO for BHBs from Motta et al. $(2014 \mathrm{a}$ b). Therefore at $\mathrm{x}<20 \mathrm{~Hz}$ the $\mathrm{y}$ axis includes a broad component, and at $x>100 \mathrm{~Hz}$ it includes the lower $\mathrm{kHz}$ QPO. The $20-100 \mathrm{~Hz}$ interval 


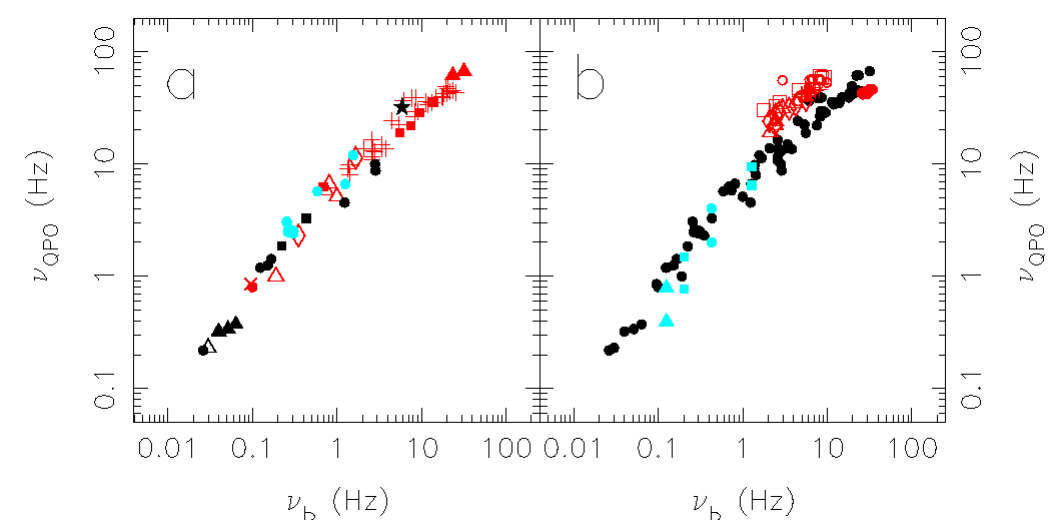

Figure 1.17 Frequency of type-C QPO and HBO vs. break frequency for a sample of NS and BH systems. Left panel: BHB in black, atoll sources in red and accreting millisecond pulsars in cyan. Panel: all points from the left panel in black, $Z$ sources in red and sources with more than one QPO is observed in cyan. (From Wijnands and van der Klis, 1999).

is covered by points from the anomalous Z-source Cir X-1, which bridges the gap in the plot. Although there seems to be a parallel correlation at very high frequencies, the correlation is very well defined and links not only $\mathrm{BH}$ and NS sources, but also broad and narrow components, suggesting that the broad features observed in the hard state mark the same physical frequencies as $\mathrm{kHz}$ QPOs (and HFQPOs) in softer states. Interestingly, an extension of this correlation by three more orders of magnitude at low frequencies has been presented when adding the frequencies of dwarf-nova oscillations and quasi-periodic oscillations observed in the optical band from cataclysmic variables (Warner et al. 2003). The extension of the correlation looks impressive, although it is not clear how to relate the signals observed in a different band from white dwarf systems, where the inner region of the accretion flow cannot exist due to the physical size of the compact star (more below).

As presented above, at low frequencies NS binaries feature three 'flavors' of QPOs: HBOs, NBOs, and FBOs and three are the 'flavors' of BH QPOs: type A,B, and C. We have seen compelling evidence that links HBOs and type-Cs. Casella et al. (2005) compared in detail the QPO types and suggested a one-to-one correspondence between them, with NBOs corresponding to type-B and HBOs to type-C. More work is needed to build on these similarities. Motta et al. (2011) on the basis of the analysis of a large sample of observations of GX 339-4 suggested the possibility that type-A QPOs are high-frequency extensions of type-C QPOs. 


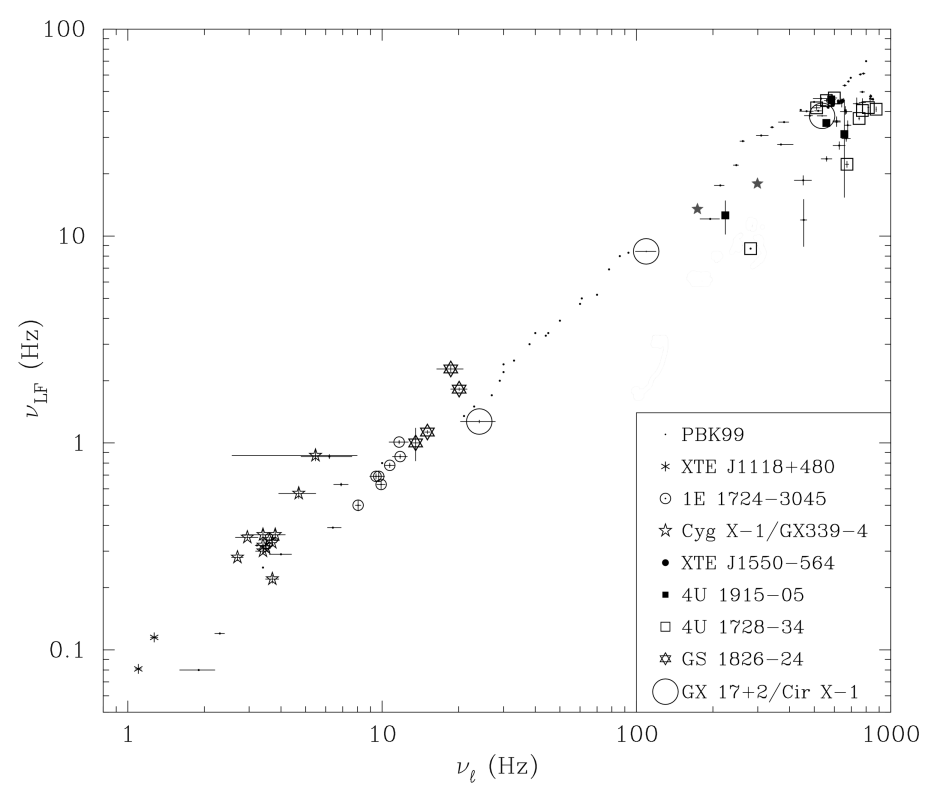

Figure 1.18 PB correlation as published by Belloni et al. (2002), with HFQPO points removed as many of them were later found not to be significant. Two points were added (five-point stars in the upper right), corresponding to the two simultaneous detections of type-C and HFQPO analysed in Motta et al. (2014a b).

Of course it is natural to explore the possibility that the two $\mathrm{kHz}$ QPOs and the two HFQPOs are the same signal observed in the vicinity of neutron stars and BHs respectively. The comparison is not simple, as very few HFQPO have been detected until now. There are clear phenomenological differences: $\mathrm{kHz}$ QPOs are observed to span a rather broad range of centroid frequencies, while the frequencies HFQPOs appear to vary only slightly if they vary at all. Moreover, the fact that $\mathrm{kHz}$ QPOs have been observed frequently and HFQPOs have not, implies that the typical fractional RMS of the latter must be lower. Until new observations with more sensitive instruments are available, it is difficult to make a full comparison.

\subsubsection{Mass measurements from $X$-rays}

Spectral analysis, whether from the continuum emission or from fluorescence lines, does not yield a mass for the compact object, which must be assumed on the basis of optical observations. Adding timing information can deliver this information, but of course a model must be assumed. The problem with past and current observatories is that they were optimised for either 
timing or high-resolution spectral analysis, which means that coordinated observations are needed, introducing further complication. A comparison was attempted on the NS binary $4 \mathrm{U}$ 1636-53 with simultaneous observations with RXTE and XMM-Newton (Sanna et al., 2014). The inner disk radius obtained by the fitting of a relativistically distorted profile to the iron emission line was combined with the information from the upper $\mathrm{kHz}$ QPO under the assumption (see below) that it represents the Keplerian frequency at the same radius. Six separate couples of simultaneous observations yielded inconsistent results on the mass, indicating that either one of the model is not correct or the two signatures do not originate from the same radius.

Fast time variability offers a potentially unique possibility for the study of effects of General Relativity in the strong-field regime and to discriminate the nature of the compact object. The information from timing signals like QPO centroid frequencies is essentially model-independent and must originate in the innermost regions of the accretion flow. In the recent years, several models have been proposed for the interpretation of these signals and almost all of them include the presence of frequencies from General Relativity. In particular, in almost all cases the highest detected feature corresponds to the Keplerian frequency at a certain radius, which can be the ISCO or larger (see above). Ideally, a successful model must be able to apply to both the NS and $\mathrm{BH}$ cases and explain the main observable facts outlined in the previous sections. One particular model (called relativistic precession model, hereafter RPM) has received particular attention. The model identifies the three observed frequencies (type-C and HFQPOs for BH binaries, $\mathrm{HBO}$ and $\mathrm{kHz}$ QPOs for neutron star binaries) to a combination of fundamental frequencies set by General Relativity. The low-frequency peaks are identified with the nodal precession frequency (Lense-Thirring), while the high frequency peaks would be the periastron precession frequency and the Keplerian frequency, all corresponding to the same orbit, which identifies a 'special' radius in the accretion flow (see Stella and Vietri, 1998, 1999, Stella et al. 1999). This model has the advantage of being extremely simple, relying on basic relativistic frequencies, and at same time with the disadvantage of being extremely simple, as it associates the frequencies to a special radius, but does not address the issues of how the oscillation is produced and why that (variable) radius is special (it cannot be the ISCO, as the two highest frequencies are identical at ISCO.

Originally applied to a sample of pairs of $\mathrm{kHz}$ QPOs, this model did not provide a precise fit, but provided frequencies in the correct range and with roughly the correct dependence (see the left panel in Figure 1.19 and Stella and Vietri, 1999, Boutloukos et al., 2006). Applied to Figure 1.19 
(where accreting millisecond pulsars are not included), the model makes three predictions, all of which observed: that the difference $\Delta \nu$ between the centroids of $\mathrm{kHz}$ QPOs must increase at low frequencies, decrease at high frequencies and have a maximum around $350-400 \mathrm{~Hz}$. The increase part is covered only by data from Cir X-1, a rather anomalous Z source (Boutloukos et al., 2006). The decrease part is more scattered, as expected if not all NS have the same mass, although the source with the highest statistics, Sco X-1, shows a decrease, steeper than expected (see Stella and Vietri, 1999). Notice that a more direct way to examine the relation between the frequencies of the two $\mathrm{kHz}$ QPO peaks is plotting one versus the other (see Figure 1.5 . where the RPM prediction for $2 \mathrm{M}_{\odot}$ is shown). In this plot, Cir X-1 and Sco X-1 are marked separately, as are accreting millisecond X-ray pulsars, which are systematically below the correlation and can be brought back to it only by multiplying both frequencies by a factor 1.5 (see Section 1.2.3). The RPM was found also to interpret naturally the PBK correlation, which also contains very broad features (Stella et al., 1999), although it would not be applicable to the white-dwarf case because of the size of the compact star being too large.

Within the RPM there is no direct dependence of $\mathrm{kHz}$ QPOs from the spin of the NS (although the model lines would be slightly modified by rotation) and indeed there is evidence that the spin does not have influence (see Section 1.2.3): Figure 1.19 (extended from that in Méndez and Belloni, 2007) shows the distribution of all published $\Delta \nu$ values (again without millisecond accreting pulsars) with a simple Gaussian fit, indicating that the typical $\Delta \nu$ value is around $300 \mathrm{~Hz}$. Why accreting millisecond pulsars, the only systems where we directly observe the pulsation, are shifted by a factor 1.5 is unclear, although also considering correlations involving the HBO suggest that both $\mathrm{kHz}$ peaks are shifted, but not the HBO (see van Straaten et al., 2005 , Linares et al. 2005).

To test the validity of the model and the relation to the spin frequency one needs an extreme source, either very slow or very fast. In 2010, an accreting accretion-powered pulsar with a rotation frequency of $11 \mathrm{~Hz}$ was discovered in outburst (Bordas et al., 2010). Unfortunately, only one $\mathrm{kHz}$ QPO was observed and it was not possible to discriminate between the (in)dependence on $\operatorname{spin}(\Delta \nu$ was expected to be $\sim 11 \mathrm{~Hz}$ or $\sim 300 \mathrm{~Hz}$ in the two scenarios). However a low-frequency QPO was observed in six observations, moving in the range $35-50 \mathrm{~Hz}$, which was identified as a $\mathrm{HBO}$ because of its intrinsic properties and positioning in the correlation diagrams Altamirano et al. 2012). Together with the observed $\mathrm{kHz}$ QPO, this results to be fast 

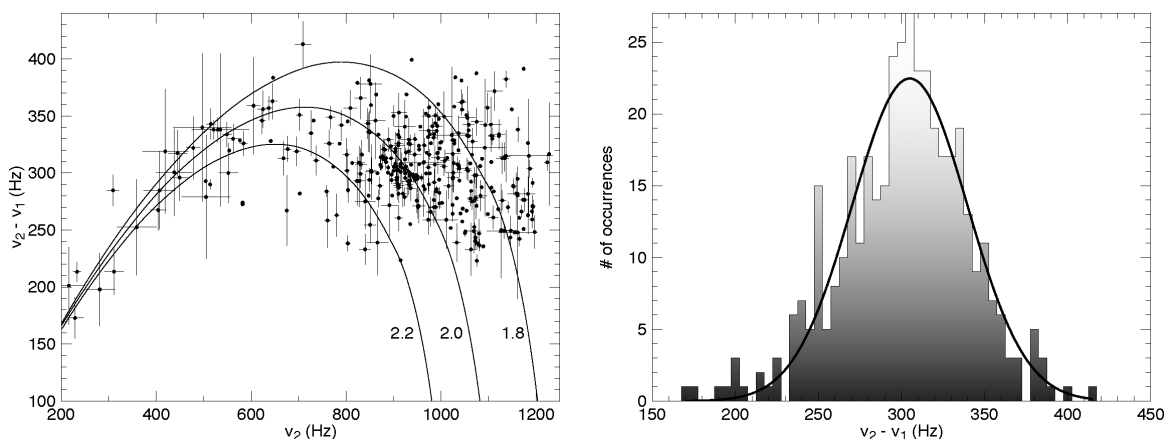

Figure 1.19 Left panel: plot of the difference $\Delta \nu$ between the centroid frequencies of $\mathrm{kHz}$ QPO pairs as a function to the centroid of the upper peak (after Stella and Vietri, 1999, Boutloukos et al., 2006) for all RXTE detections in the literature from NS LMXBs (excluding accreting millisecond pulsars). The lines are predictions from the RPM for three NS masses (indicated in solar masses). Right panel: distribution of all $\Delta \nu$ values on the $\mathrm{Y}$ axis of the plot in the left panel (after Méndez and Belloni, 2007). The line is a Gaussian fit with centroid $\sim 305 \mathrm{~Hz}$.

to be associated to with Lense-Thirring frequency at the same radius. More observations will be needed to firmly confirm the lack of association.

The curves in the left panel of Figure 1.19 can be calculated also for BHs, i.e. higher masses, and obviously would lie to lower frequencies, merging with the others at their low end. For a $10 \mathrm{M}_{\odot} \mathrm{BH}$, the curve would peak around $120 \mathrm{~Hz}$. Of course for low-frequency broad features the model does not allow to discriminate the compact object, but notice that Figure 1.19 indicates that the RPM identifies all sources excluding Cir X-1 as NSs.

As mentioned above, there are only a few detections of HFQPOs and fewer detections of pairs of them. Moreover, the presence of HFQPOs seems to be mutually exclusive with that of type-C QPOs. However, there is a single observation of GRO J1655-40, the only RXTE observation in twelve years of operations, in which all three frequencies (one type-C and two HFQPOs) have been observed (see Strohmayera, 2001, and Figure 1.20). From these three values, measured with considerable precision, it has been possible to apply the RPM relations and obtain three physical parameters: the radius $R$ of the orbit to which they are related, the mass $M$ and the spin $a$ of the compact object (Motta et al., 2014a). The derived parameters are $R=5.677 \pm 0.035 \mathrm{r}_{g}, M=5.307 \pm 0.066 \mathrm{M}_{\odot}$, and $a=0.286 \pm 0.003$. Although with a small error bar, the derived mass is compatible with the precise dynamical measurement of $M=5.4 \pm 0.4 \mathrm{M}_{\odot}$ (Beer and Podsiadlowski, 2002). Being based only on one detection, this result will have to be corroborated 


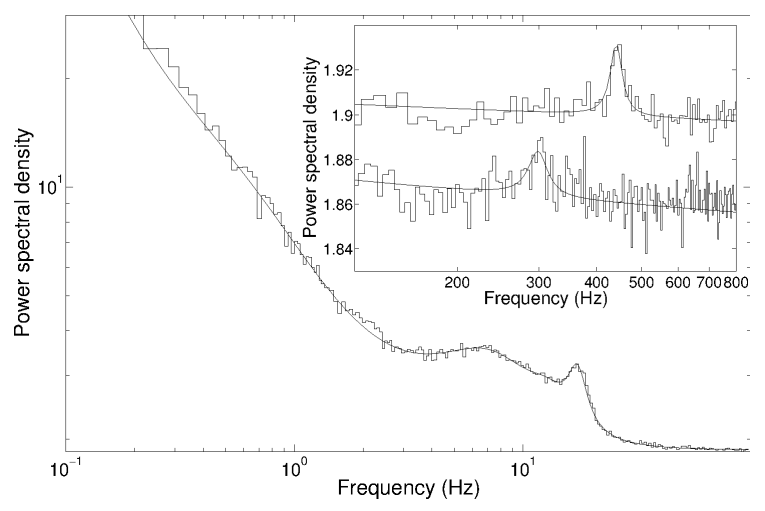

Figure 1.20 Power density spectrum of the only $R X T E$ observation of a $\mathrm{BHB}$ with a triplet of QPOs. The main panel shows the $18 \mathrm{~Hz}$ type-C QPO, the inset shows the high-frequency part in two separate energy bands to highlight the two HFQPOs. (From Motta et al., 2014a).

by more observations, but in principle this would constitute the first direct evidence of the presence of a black hole in an X-ray binary, as well as a precise measurement of its spin. In addition, since $a$ and $M$ define fully the system, it is possible to measure the radius of the ISCO and therefore the maximum values that the frequencies of the three peaks can reach. While the few detections of HFQPOs are all around the same values, a large number of type-C frequencies were measured throughout the two outbursts of this system, and their frequencies are distributed between $0.1 \mathrm{~Hz}$ and $28 \mathrm{~Hz}$, the latter only marginally above the maximum value allowed by the model (red points in Figure 1.21, see Motta et al., 2014a). If confirmed by more data, this constitutes a direct measurement of the presence of the ISCO. Finally, adding all detections of broad components in the PDS, identified as $\nu_{\ell}$ and $\nu_{u}$ (black points in Figure 1.21, the PBK correlation), they line along the prediction of the model.

The same analysis can be done on the only observation of XTE J1550-564 that shows a type-C and a single HFQPO (Motta et al., 2014b). Here the third parameter needed to solve the system of GR equations cannot be the second HFQPO, so the mass measured from optical observations was used. The same analysis led to the measurement of a spin of $a=0.34 \pm 0.01$, with all type-C frequencies being below the maximum ISCO values and the broad components fitting the model.

The identification of the physical origin of the observed frequencies is not sufficient for a solid model and a modulation process is necessary. A promising model has been proposed for the $\mathrm{BH}$ case, based on a thermal disk 


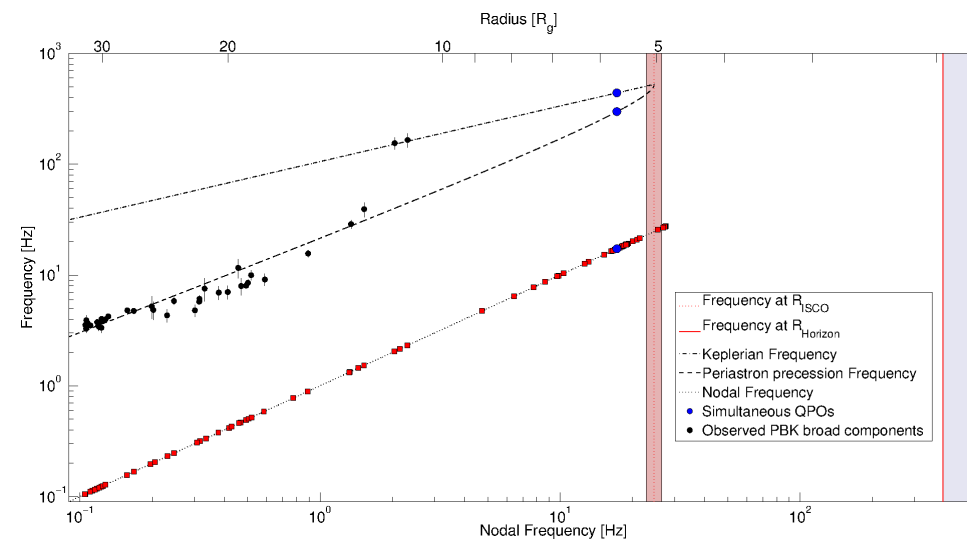

Figure 1.21 Frequency correlations in the full sample of RXTE observations of GRO J1655-40. The red points represent type-C vs. type-C, therefore lie on a 1:1 line. The three blue points are the frequencies of the triplet of QPOs used to determine mass and spin of the $\mathrm{BH}$. The black points are the $\nu_{\ell}$ and $\nu_{u}$ frequencies as a function of type-C QPO ( $\nu_{\ell}$ vs. type-C is the PBK correlation). The lines are the dependencies predicted by the RPM for Keplerian, periastron precession and nodal precession (from top to bottom) for the best fit parameters from the blue points. The red band indicates the maximum nodal frequency set by the presence of an ISCO in the system. (From Motta et al., 2014a).

with an inner truncation radius, below which the accretion flow is geometrically thick model consistent with spectral models (see e.g. Poutanen et al., 1997). This model interprets type-C QPO as arising from the frame-dragging precession of the inner flow, where the flux is modulated by effects like selfoccultation, relativistic effects and changes in projected area. Although each radius has a different precession frequency, the overall result is an oscillation at a frequency intermediate between those at the outer and inner boundary of the geometrically thick precessing region (Ingram et al., 2009), with the broad-band noise arising from propagation effects (see above and Ingram and Done, 2010, 2011; Ingram and van der Klis, 2013). This model does not address (yet) the high-frequency oscillations nor the case of NSs, but it is being investigated further through the search and detection of modulation of the effects of the inner-flow precession on the properties of the radiation reflected by the geometrically thin accretion disk. A modulation at the QPO period of the iron line due to reflection has been detected, strengthening the applicability of the model (Ingram et al., 2016).

Finally, an alternative model for low-frequency QPOs, the transition layer model, interprets type-C QPOs as viscous magneto-acoustic oscillations of 
a spherical transition layer between the Keplerian flow and a sub-Keplerian region near the BH (Titarchuk and Fiorito, 2004). This model predicts a relationship between the photon index of the hard spectral component and the frequency of the type-C QPO, which is used to measure the mass of the BH (see Gliozzi et al., 2011).

\subsection{Conclusions}

In the sections above, I have presented the current standpoint in a selected number of topics regarding X-ray emission from BH and NS binaries. The past two decades have seen a dramatic increase in our knowledge of the process of accretion onto compact objects. We now know that the presence of collimated relativistic jets and powerful wind outflows from X-ray binaries, which I did not touch here, is impossible to ignore if we want to understand the physics of accretion. At the same time, high-resolution spectral information, long-term coverage of transient systems and high-sensitivity timing analysis have proven to be essential, in particular when combined. With current instruments, we now have the tools to start disentangling the effects of accretion that are in common between NS and BH sources and those connected to the nature of the compact object. This will allow us to use these powerful sources to study both astrophysics and basic physics, making the best use of these cosmic laboratories.

\subsection{Acknowledgements}

I thank S. Motta, M. Méndez, D. Altamirano and L. Stella or useful discussions and the University of Southampton for providing more than once the ideal environment to write this chapter. I also thank the editors for their work on the Winter School and on the book, and last but not least for bearing with me. Finally, I would like to thank Dr. M. Mantica for getting rid of the only transient QPO signal that I was definitely not interested in.

\section{References}

Abbott, B. P., Abbott, R., Abbott, T. D., Abernathy, M. R., Acernese, F., Ackley, K., Adams, C., Adams, T., Addesso, P., Adhikari, R. X., and et al. 2016. Observation of Gravitational Waves from a Binary Black Hole Merger. Physical Review Letters, 116(6), 061102.

Abramowicz, M. A., and Kluźniak, W. 2001. A precise determination of black hole spin in GRO J1655-40. A\&A, 374(Aug.), L19-L20.

Abramowicz, M. A., Kluźniak, W., and Lasota, J.-P. 2002. No observational proof of the black-hole event-horizon. A\&A, 396(Dec.), L31-L34. 
Altamirano, D., and Belloni, T. 2012. Discovery of High-frequency Quasi-periodic Oscillations in the Black Hole Candidate IGR J17091-3624. ApJ, 747(Mar.), L4.

Altamirano, D., Ingram, A., van der Klis, M., Wijnands, R., Linares, M., and Homan, J. 2012. Low-frequency Quasi-periodic Oscillation from the $11 \mathrm{~Hz}$ Accreting Pulsar in Terzan 5: Not Frame Dragging. ApJ, 759(Nov.), L20.

Barret, D., Olive, J.-F., and Miller, M. C. 2005a. An abrupt drop in the coherence of the lower $\mathrm{kHz}$ quasi-periodic oscillations in $4 \mathrm{U}$ 1636-536. MNRAS, 361(Aug.), 855-860.

Barret, D., Kluźniak, W., Olive, J. F., Paltani, S., and Skinner, G. K. 2005b. On the high coherence of $\mathrm{kHz}$ quasi-periodic oscillations. MNRAS, 357(Mar.), $1288-1294$.

Barret, D., Olive, J.-F., and Miller, M. C. 2006. The coherence of kilohertz quasiperiodic oscillations in the X-rays from accreting neutron stars. MNRAS, 370(Aug.), 1140-1146.

Barret, D., Olive, J.-F., and Miller, M. C. 2007. Supporting evidence for the signature of the innermost stable circular orbit in Rossi X-ray data from $4 \mathrm{U}$ 1636-536. MNRAS, 376(Apr.), 1139-1144.

Beer, M. E., and Podsiadlowski, P. 2002. The quiescent light curve and the evolutionary state of GRO J1655-40. MNRAS, 331(Mar.), 351-360.

Belloni, T., Mendez, M., van der Klis, M., Hasinger, G., Lewin, W. H. G., and van Paradijs, J. 1996. An Intermediate State of Cygnus X-1. ApJ, 472(Dec.), L107.

Belloni, T., van der Klis, M., Lewin, W. H. G., van Paradijs, J., Dotani, T., Mitsuda, K., and Miyamoto, S. 1997. Energy dependence in the quasi-periodic oscillations and noise of black hole candidates in the very high state. A\&A, 322(June), 857-867.

Belloni, T., Méndez, M., and Sánchez-Fernández, C. 2001. The high-frequency QPOs in GRS 1915+105. A\&A, 372(June), 551-556.

Belloni, T., Psaltis, D., and van der Klis, M. 2002. A Unified Description of the Timing Features of Accreting X-Ray Binaries. ApJ, 572(June), 392-406.

Belloni, T., Homan, J., Casella, P., van der Klis, M., Nespoli, E., Lewin, W. H. G., Miller, J. M., and Méndez, M. 2005. The evolution of the timing properties of the black-hole transient GX 339-4 during its 2002/2003 outburst. A\&A, 440(Sept.), 207-222.

Belloni, T., Méndez, M., and Homan, J. 2007a. On the kHz QPO frequency correlations in bright neutron star X-ray binaries. MNRAS, 376(Apr.), 1133-1138.

Belloni, T., Homan, J., Motta, S., Ratti, E., and Méndez, M. 2007b. Rossi XTE monitoring of 4U1636-53 - I. Long-term evolution and $\mathrm{kHz}$ quasi-periodic oscillations. MNRAS, 379(July), 247-252.

Belloni, T. M. 2010 (Mar.). States and Transitions in Black Hole Binaries. Page 53 of: Belloni, T. (ed), Lecture Notes in Physics, Berlin Springer Verlag. Lecture Notes in Physics, Berlin Springer Verlag, vol. 794.

Belloni, T. M., and Altamirano, D. 2013a. Discovery of a $34 \mathrm{~Hz}$ quasi-periodic oscillation in the X-ray emission of GRS 1915+105. MNRAS, 432(June), 19-22.

Belloni, T. M., and Altamirano, D. 2013b. High-frequency quasi-periodic oscillations from GRS 1915+105. MNRAS, 432(June), 10-18. 
Belloni, T. M., and Motta, S. E. 2016. Transient Black Hole Binaries. Page 61 of: Bambi, C. (ed), Astrophysics of Black Holes: From Fundamental Aspects to Latest Developments. Astrophysics and Space Science Library, vol. 440.

Belloni, T. M., Motta, S. E., and Muñoz-Darias, T. 2011. Black hole transients. Bulletin of the Astronomical Society of India, 39(Sept.), 409-428.

Belloni, T. M., Sanna, A., and Méndez, M. 2012. High-frequency quasi-periodic oscillations in black hole binaries. MNRAS, 426(Nov.), 1701-1709.

Bordas, P., Kuulkers, E., Alfonso-Garzón, J., Beckmann, V., Bird, T., Chenevez, S. B. J., Courvoisier, T., Del Santo, M., Domingo, A., Ebisawa, K., Ferrigno, C., Jonker, P., Kretschmar, P., Markwardt, C., Oosterbroek, T., Paizis, A., Pottschmidt, K., Sánchez-Fernández, C., and Wijnands, R. 2010. A hard X-ray transient in the direction of Terzan 5 detected by INTEGRAL. The Astronomer's Telegram, 2919(Oct.).

Boutloukos, S., van der Klis, M., Altamirano, D., Klein-Wolt, M., Wijnands, R., Jonker, P. G., and Fender, R. P. 2006. Discovery of Twin kHz QPOs in the Peculiar X-Ray Binary Circinus X-1. ApJ, 653(Dec.), 1435-1444.

Brown, E. F., and Cumming, A. 2009. Mapping Crustal Heating with the Cooling Light Curves of Quasi-Persistent Transients. ApJ, 698(June), 1020-1032.

Bu, Q.-c., Chen, L., Li, Z.-s., Qu, J.-l., Belloni, T. M., and Zhang, L. 2015. Correlations in Horizontal Branch Oscillations and Break Components in XTE J1701-462 and GX 17+2. ApJ, 799(Jan.), 2.

Burgay, M., Kramer, M., and McLaughlin, M. A. 2014. The Double Pulsar J07373039A/B: a decade of surprises. Bulletin of the Astronomical Society of India, 42(Sept.), 101-119.

Burke, M. J., Gilfanov, M., and Sunyaev, R. 2016. A dichotomy between the hard state spectral properties of black hole and neutron star X-ray binaries. MNRAS, Oct.

Casares, J., and Jonker, P. G. 2014. Mass Measurements of Stellar and IntermediateMass Black Holes. Space Sci. Rev., 183(Sept.), 223-252.

Casella, P., Belloni, T., Homan, J., and Stella, L. 2004. A study of the low-frequency quasi-periodic oscillations in the X-ray light curves of the black hole candidate; XTE J1859+226. A\&A, 426(Nov.), 587-600.

Casella, P., Belloni, T., and Stella, L. 2005. The ABC of Low-Frequency Quasiperiodic Oscillations in Black Hole Candidates: Analogies with Z Sources. ApJ, 629(Aug.), 403-407.

Davis, S. W., Blaes, O. M., Hubeny, I., and Turner, N. J. 2005. Relativistic Accretion Disk Models of High-State Black Hole X-Ray Binary Spectra. ApJ, 621(Mar.), 372-387.

de Avellar, M. G. B., Méndez, M., Sanna, A., and Horvath, J. E. 2013. Time lags of the kilohertz quasi-periodic oscillations in the low-mass X-ray binaries $4 \mathrm{U}$ 1608-52 and 4U 1636-53. MNRAS, 433(Aug.), 3453-3463.

Degenaar, N., Miller, J. M., Chakrabarty, D., Harrison, F. A., Kara, E., and Fabian, A. C. 2015. A NuSTAR observation of disc reflection from close to the neutron star in 4U 1608-52. MNRAS, 451(July), L85-L89.

Del Santo, M., Malzac, J., Jourdain, E., Belloni, T., and Ubertini, P. 2008. Spectral variability of GX339-4 in a hard-to-soft state transition. MNRAS, 390(Oct.), $227-234$.

Di Salvo, T., Iaria, R., Matranga, M., Burderi, L., D’Aí, A., Egron, E., Papitto, A., Riggio, A., Robba, N. R., and Ueda, Y. 2015. Suzaku broad-band spectrum 
of $4 \mathrm{U}$ 1705-44: probing the reflection component in the hard state. MNRAS, 449(May), 2794-2802.

Dinçer, T., Kalemci, E., Tomsick, J. A., Buxton, M. M., and Bailyn, C. D. 2014. Complete Multiwavelength Evolution of Galactic Black Hole Transients during Outburst Decay. II. Compact Jets and X-Ray Variability Properties. ApJ, 795(Nov.), 74.

Farinelli, R., Titarchuk, L., Paizis, A., and Frontera, F. 2008. A New Comptonization Model for Weakly Magnetized, Accreting Neutron Stars in Low-Mass X-Ray Binaries. ApJ, 680(June), 602-614.

Garcia, M. R., McClintock, J. E., Narayan, R., Callanan, P., Barret, D., and Murray, S. S. 2001. New Evidence for Black Hole Event Horizons from Chandra. ApJ, 553(May), L47-L50.

Gierliński, M., and Done, C. 2002. The X-ray spectrum of the atoll source $4 \mathrm{U}$ 1608-52. MNRAS, 337(Dec.), 1373-1380.

Gilfanov, M. 2010 (Mar.). X-Ray Emission from Black-Hole Binaries. Page 17 of: Belloni, T. (ed), Lecture Notes in Physics, Berlin Springer Verlag. Lecture Notes in Physics, Berlin Springer Verlag, vol. 794.

Gliozzi, M., Titarchuk, L., Satyapal, S., Price, D., and Jang, I. 2011. Testing a Scaleindependent Method to Measure the Mass of Black Holes. ApJ, 735(July), 16.

Grove, J. E., Johnson, W. N., Kroeger, R. A., McNaron-Brown, K., Skibo, J. G., and Phlips, B. F. 1998. Gamma-Ray Spectral States of Galactic Black Hole Candidates. ApJ, 500(June), 899-908.

Hasinger, G., and van der Klis, M. 1989. Two patterns of correlated X-ray timing and spectral behaviour in low-mass X-ray binaries. A\&A, 225(Nov.), 79-96.

Heil, L. M., Vaughan, S., and Uttley, P. 2012. The ubiquity of the rms-flux relation in black hole X-ray binaries. MNRAS, 422(May), 2620-2631.

Homan, J., Wijnands, R., van der Klis, M., Belloni, T., van Paradijs, J., Klein-Wolt, M., Fender, R., and Méndez, M. 2001. Correlated X-Ray Spectral and Timing Behavior of the Black Hole Candidate XTE J1550-564: A New Interpretation of Black Hole States. ApJS, 132(Feb.), 377-402.

Homan, J., van der Klis, M., Jonker, P. G., Wijnands, R., Kuulkers, E., Méndez, M., and Lewin, W. H. G. 2002. RXTE Observations of the Neutron Star Low-Mass X-Ray Binary GX 17+2: Correlated X-Ray Spectral and Timing Behavior. ApJ, 568(Apr.), 878-900.

Homan, J., Klein-Wolt, M., Rossi, S., Miller, J. M., Wijnands, R., Belloni, T., van der Klis, M., and Lewin, W. H. G. 2003. High-Frequency Quasi-periodic Oscillations in the Black Hole X-Ray Transient XTE J1650-500. ApJ, 586(Apr.), $1262-1267$.

Homan, J., Miller, J. M., Wijnands, R., van der Klis, M., Belloni, T., Steeghs, D., and Lewin, W. H. G. 2005a. High- and Low-Frequency Quasi-periodic Oscillations in the X-Ray Light Curves of the Black Hole Transient H1743322. ApJ, 623(Apr.), 383-391.

Homan, J., Buxton, M., Markoff, S., Bailyn, C. D., Nespoli, E., and Belloni, T. 2005b. Multiwavelength Observations of the 2002 Outburst of GX 339-4: Two Patterns of X-Ray-Optical/Near-Infrared Behavior. ApJ, 624(May), 295-306.

Homan, J., van der Klis, M., Wijnands, R., Belloni, T., Fender, R., Klein-Wolt, M., Casella, P., Méndez, M., Gallo, E., Lewin, W. H. G., and Gehrels, N. 2007. Rossi X-Ray Timing Explorer Observations of the First Transient Z 
Source XTE J1701-462: Shedding New Light on Mass Accretion in Luminous Neutron Star X-Ray Binaries. ApJ, 656(Feb.), 420-430.

Homan, J., van der Klis, M., Fridriksson, J. K., Remillard, R. A., Wijnands, R., Méndez, M., Lin, D., Altamirano, D., Casella, P., Belloni, T. M., and Lewin, W. H. G. 2010. XTE J1701-462 and Its Implications for the Nature of Subclasses in Low-magnetic-field Neutron Star Low-mass X-ray Binaries. ApJ, 719(Aug.), 201-212.

Ingram, A., and Done, C. 2010. A physical interpretation of the variability power spectral components in accreting neutron stars. MNRAS, 405(July), 24472452.

Ingram, A., and Done, C. 2011. A physical model for the continuum variability and quasi-periodic oscillation in accreting black holes. MNRAS, 415(Aug.), $2323-2335$.

Ingram, A., and van der Klis, M. 2013. An exact analytic treatment of propagating mass accretion rate fluctuations in X-ray binaries. MNRAS, 434(Sept.), 14761485 .

Ingram, A., Done, C., and Fragile, P. C. 2009. Low-frequency quasi-periodic oscillations spectra and Lense-Thirring precession. MNRAS, 397(July), L101-L105.

Ingram, A., van der Klis, M., Middleton, M., Done, C., Altamirano, D., Heil, L., Uttley, P., and Axelsson, M. 2016. A quasi-periodic modulation of the iron line centroid energy in the black hole binary H1743-322. MNRAS, 461(Sept.), 1967-1980.

Joinet, A., Kalemci, E., and Senziani, F. 2008. Hard X-Ray Emission of the Microquasar GRO J1655-40 during the Rise of Its 2005 Outburst. ApJ, 679(May), $655-663$

Kalemci, E., Dinçer, T., Tomsick, J. A., Buxton, M. M., Bailyn, C. D., and Chun, Y. Y. 2013. Complete Multiwavelength Evolution of Galactic Black Hole Transients during Outburst Decay. I. Conditions for "Compact" Jet Formation. ApJ, 779(Dec.), 95.

Klein-Wolt, M., Homan, J., and van der Klis, M. 2004. High frequency features in the 1998 outburst of 4U 1630-47. Nuclear Physics B Proceedings Supplements, 132(June), 381-386.

Kolehmainen, M., Done, C., and Díaz Trigo, M. 2014. The soft component and the iron line as signatures of the disc inner radius in Galactic black hole binaries. MNRAS, 437(Jan.), 316-326.

Kong, A. K. H., McClintock, J. E., Garcia, M. R., Murray, S. S., and Barret, D. 2002. The X-Ray Spectra of Black Hole X-Ray Novae in Quiescence as Measured by Chandra. ApJ, 570(May), 277-286.

Kylafis, N. D., and Belloni, T. M. 2015. Accretion and ejection in black-hole X-ray transients. A\&A, 574(Feb.), A133.

Lasota, J.-P. 2016. Black Hole Accretion Discs. Page 1 of: Bambi, C. (ed), Astrophysics of Black Holes: From Fundamental Aspects to Latest Developments. Astrophysics and Space Science Library, vol. 440.

Lin, D., Remillard, R. A., and Homan, J. 2007. Evaluating Spectral Models and the X-Ray States of Neutron Star X-Ray Transients. ApJ, 667(Oct.), 1073-1086.

Lin, D., Remillard, R. A., and Homan, J. 2009. Spectral States of XTE J1701 462: Link Between Z and Atoll Sources. ApJ, 696(May), 1257-1277.

Lin, D., Remillard, R. A., Homan, J., and Barret, D. 2012. The Spectral Evolution along the Z Track of the Bright Neutron Star X-Ray Binary GX 17+2. ApJ, 756(Sept.), 34 . 
Linares, M., van der Klis, M., Altamirano, D., and Markwardt, C. B. 2005. Discovery of Kilohertz Quasi-periodic Oscillations and Shifted Frequency Correlations in the Accreting Millisecond Pulsar XTE J1807-294. ApJ, 634(Dec.), $1250-1260$.

Lyubarskii, Y. E. 1997. Flicker noise in accretion discs. MNRAS, 292(Dec.), 679.

Markoff, S. 2010 (Mar.). From Multiwavelength to Mass Scaling: Accretion and Ejection in Microquasars and AGN. Page 143 of: Belloni, T. (ed), Lecture Notes in Physics, Berlin Springer Verlag. Lecture Notes in Physics, Berlin Springer Verlag, vol. 794.

Markoff, S., Nowak, M. A., and Wilms, J. 2005. Going with the Flow: Can the Base of Jets Subsume the Role of Compact Accretion Disk Coronae? ApJ, 635(Dec.), 1203-1216.

McClintock, J. E., Narayan, R., Garcia, M. R., Orosz, J. A., Remillard, R. A., and Murray, S. S. 2003. Multiwavelength Spectrum of the Black Hole XTE J1118+480 in Quiescence. ApJ, 593(Aug.), 435-451.

Méndez, M. 2006. On the maximum amplitude and coherence of the kilohertz quasi-periodic oscillations in low-mass X-ray binaries. MNRAS, 371(Oct.), $1925-1938$.

Méndez, M., and Belloni, T. 2007. Is there a link between the neutron-star spin and the frequency of the kilohertz quasi-periodic oscillations? MNRAS, 381(Oct.), 790-796.

Méndez, M., and van der Klis, M. 1997. The EXOSAT Data on GX 339-4: Further Evidence for an "Intermediate" State. ApJ, 479(Apr.), 926-932.

Méndez, M., van der Klis, M., Wijnands, R., Ford, E. C., van Paradijs, J., and Vaughan, B. A. 1998. Kilohertz Quasi-periodic Oscillation Peak Separation Is Not Constant in the Atoll Source 4U 1608-52. ApJ, 505(Sept.), L23-L26.

Méndez, M., Altamirano, D., Belloni, T., and Sanna, A. 2013. The phase lags of high-frequency quasi-periodic oscillations in four black hole candidates. MNRAS, 435(Nov.), 2132-2140.

Menou, K., Esin, A. A., Narayan, R., Garcia, M. R., Lasota, J.-P., and McClintock, J. E. 1999. Black Hole and Neutron Star Transients in Quiescence. ApJ, 520(July), 276-291.

Middleton, M. 2016. Black Hole Spin: Theory and Observation. Page 99 of: Bambi, C. (ed), Astrophysics of Black Holes: From Fundamental Aspects to Latest Developments. Astrophysics and Space Science Library, vol. 440.

Migliari, S., van der Klis, M., and Fender, R. P. 2003. Evidence of a decrease of $\mathrm{kHz}$ quasi-periodic oscillation peak separation towards low frequencies in $4 \mathrm{U}$ 1728-34 (GX 354-0). MNRAS, 345(Nov.), L35-L39.

Migliari, S., Miller-Jones, J. C. A., Fender, R. P., Homan, J., Di Salvo, T., Rothschild, R. E., Rupen, M. P., Tomsick, J. A., Wijnands, R., and van der Klis, M. 2007. Linking Jet Emission, X-Ray States, and Hard X-Ray Tails in the Neutron Star X-Ray Binary GX 17+2. ApJ, 671(Dec.), 706-712.

Miller, J. M. 2007. Relativistic X-Ray Lines from the Inner Accretion Disks Around Black Holes. ARA\&A, 45(Sept.), 441-479.

Miller, J. M., Wijnands, R., Homan, J., Belloni, T., Pooley, D., Corbel, S., Kouveliotou, C., van der Klis, M., and Lewin, W. H. G. 2001. High-Frequency Quasi-Periodic Oscillations in the 2000 Outburst of the Galactic Microquasar XTE J1550-564. ApJ, 563(Dec.), 928-933.

Mitsuda, K., Inoue, H., Nakamura, N., and Tanaka, Y. 1989. Luminosity-related changes of the energy spectrum of X1608-522. PASJ, 41, 97-111. 
Miyamoto, S., Kitamoto, S., Iga, S., Negoro, H., and Terada, K. 1992. Canonical time variations of $\mathrm{X}$-rays from black hole candidates in the low-intensity state. ApJ, 391(May), L21-L24.

Miyamoto, S., Iga, S., Kitamoto, S., and Kamado, Y. 1993. Another canonical time variation of X-rays from black hole candidates in the very high flare state? ApJ, 403(Jan.), L39-L42.

Morgan, E. H., Remillard, R. A., and Greiner, J. 1997. RXTE Observations of QPOs in the Black Hole Candidate GRS 1915+105. ApJ, 482(June), 9931010.

Motta, S., Belloni, T., and Homan, J. 2009. The evolution of the high-energy cut-off in the X-ray spectrum of GX 339-4 across a hard-to-soft transition. MNRAS, 400(Dec.), 1603-1612.

Motta, S., Muñoz-Darias, T., Casella, P., Belloni, T., and Homan, J. 2011. Lowfrequency oscillations in black holes: a spectral-timing approach to the case of GX 339-4. MNRAS, 418(Dec.), 2292-2307.

Motta, S., Homan, J., Muñoz Darias, T., Casella, P., Belloni, T. M., Hiemstra, B., and Méndez, M. 2012. Discovery of two simultaneous non-harmonically related quasi-periodic oscillations in the 2005 outburst of the black hole binary GRO J1655-40. MNRAS, 427(Nov.), 595-606.

Motta, S. E. 2016. Quasi periodic oscillations in black hole binaries. Astronomische Nachrichten, 337(May), 398.

Motta, S. E., Muñoz-Darias, T., Sanna, A., Fender, R., Belloni, T., and Stella, L. 2014a. Black hole spin measurements through the relativistic precession model: XTE J1550-564. MNRAS, 439(Mar.), L65-L69.

Motta, S. E., Belloni, T. M., Stella, L., Muñoz-Darias, T., and Fender, R. 2014b. Precise mass and spin measurements for a stellar-mass black hole through X-ray timing: the case of GRO J1655-40. MNRAS, 437(Jan.), 2554-2565.

Muñoz-Darias, T., Motta, S., and Belloni, T. M. 2011. Fast variability as a tracer of accretion regimes in black hole transients. MNRAS, 410(Jan.), 679-684.

Muñoz-Darias, T., Coriat, M., Plant, D. S., Ponti, G., Fender, R. P., and Dunn, R. J. H. 2013. Inclination and relativistic effects in the outburst evolution of black hole transients. MNRAS, 432(June), 1330-1337.

Muñoz-Darias, T., Fender, R. P., Motta, S. E., and Belloni, T. M. 2014. Black holelike hysteresis and accretion states in neutron star low-mass X-ray binaries. MNRAS, 443(Oct.), 3270-3283.

Narayan, R., and Heyl, J. S. 2002. On the Lack of Type I X-Ray Bursts in Black Hole X-Ray Binaries: Evidence for the Event Horizon? ApJ, 574(Aug.), L139L142.

Narayan, R., McClintock, J. E., and Yi, I. 1996. A New Model for Black Hole Soft X-Ray Transients in Quiescence. ApJ, 457(Feb.), 821.

Nespoli, E., Belloni, T., Homan, J., Miller, J. M., Lewin, W. H. G., Méndez, M., and van der Klis, M. 2003. A transient variable $6 \mathrm{~Hz}$ QPO from GX 339-4. A\&A, 412(Dec.), 235-240.

Nowak, M. A. 2000. Are there three peaks in the power spectra of GX 339-4 and Cyg X-1? MNRAS, 318(Oct.), 361-367.

Olive, J. F., Barret, D., Boirin, L., Grindlay, J. E., Swank, J. H., and Smale, A. P. 1998. RXTE observation of the X-ray burster 1E 1724-3045. I. Timing study of the persistent X-ray emission with the PCA. A\&A, 333(May), 942-951.

Petrucci, P. O. 2008. Models of comptonization. Mem. Soc. Astron. Italiana, 79, 118. 
Petrucci, P.-O., Ferreira, J., Henri, G., and Pelletier, G. 2008. The role of the disc magnetization on the hysteresis behaviour of X-ray binaries. MNRAS, 385(Mar.), L88-L92.

Plotkin, R. M., Gallo, E., and Jonker, P. G. 2013. The X-Ray Spectral Evolution of Galactic Black Hole X-Ray Binaries toward Quiescence. ApJ, 773(Aug.), 59 .

Plotkin, R. M., Gallo, E., Markoff, S., Homan, J., Jonker, P. G., Miller-Jones, J. C. A., Russell, D. M., and Drappeau, S. 2015. Constraints on relativistic jets in quiescent black hole X-ray binaries from broad-band spectral modelling. MNRAS, 446(Feb.), 4098-4111.

Pottschmidt, K., Wilms, J., Nowak, M. A., Pooley, G. G., Gleissner, T., Heindl, W. A., Smith, D. M., Remillard, R., and Staubert, R. 2003. Long term variability of Cygnus X-1. I. X-ray spectral-temporal correlations in the hard state. A\&A, 407(Sept.), 1039-1058.

Poutanen, J., Krolik, J. H., and Ryde, F. 1997. The nature of spectral transitions in accreting black holes - The case of CYG X-1. MNRAS, 292(Nov.), L21-L25.

Psaltis, D. 2008. Probes and Tests of Strong-Field Gravity with Observations in the Electromagnetic Spectrum. Living Reviews in Relativity, 11(Dec.), 9.

Psaltis, D., Belloni, T., and van der Klis, M. 1999a. Correlations in Quasi-periodic Oscillation and Noise Frequencies among Neutron Star and Black Hole X-Ray Binaries. ApJ, 520(July), 262-270.

Psaltis, D., Wijnands, R., Homan, J., Jonker, P. G., van der Klis, M., Miller, M. C., Lamb, F. K., Kuulkers, E., van Paradijs, J., and Lewin, W. H. G. 1999b. On the Magnetospheric Beat-Frequency and Lense-Thirring Interpretations of the Horizontal-Branch Oscillation in the Z Sources. ApJ, 520(Aug.), 763-775.

Rao, F., Belloni, T., Stella, L., Zhang, S. N., and Li, T. 2010. Low-frequency Oscillations in XTE J1550-564. ApJ, 714(May), 1065-1071.

Ratti, E. M., Belloni, T. M., and Motta, S. E. 2012. On the harmonics of the lowfrequency quasi-periodic oscillation in GRS 1915+105. MNRAS, 423(June), 694-701.

Reid, M. J., McClintock, J. E., Steiner, J. F., Steeghs, D., Remillard, R. A., Dhawan, V., and Narayan, R. 2014. A Parallax Distance to the Microquasar GRS 1915+105 and a Revised Estimate of its Black Hole Mass. ApJ, 796(Nov.), 2.

Remillard, R. A., Morgan, E. H., McClintock, J. E., Bailyn, C. D., and Orosz, J. A. 1999a. RXTE Observations of 0.1-300 HZ Quasi-periodic Oscillationsin the Microquasar GRO J1655-40. ApJ, 522(Sept.), 397-412.

Remillard, R. A., McClintock, J. E., Sobczak, G. J., Bailyn, C. D., Orosz, J. A., Morgan, E. H., and Levine, A. M. 1999b. X-Ray Nova XTE J1550-564: Discovery of a Quasi-periodic Oscillation near 185 HZ. ApJ, 517(June), L127-L130.

Remillard, R. A., Muno, M. P., McClintock, J. E., and Orosz, J. A. 2002. Evidence for Harmonic Relationships in the High-Frequency Quasi-periodic Oscillations of XTE J1550-564 and GRO J1655-40. ApJ, 580(Dec.), 1030-1042.

Remillard, R. A., Lin, D., ASM Team at MIT, and NASA/GSFC. 2006a. New X-ray Transient, XTE J1701-462. The Astronomer's Telegram, 696(Jan.).

Remillard, R. A., Lin, D., Cooper, R. L., and Narayan, R. 2006b. The Rates of Type I X-Ray Bursts from Transients Observed with RXTE: Evidence for Black Hole Event Horizons. ApJ, 646(July), 407-419.

Remillard, R. A., McClintock, J. E., Orosz, J. A., and Levine, A. M. 2006c. The X-Ray Outburst of H1743-322 in 2003: High-Frequency QPOs with a 3:2 Frequency Ratio. ApJ, 637(Feb.), 1002-1009. 
Sanna, A., Méndez, M., Belloni, T., and Altamirano, D. 2012. The time derivative of the kilohertz quasi-periodic oscillations in $4 \mathrm{U}$ 1636-53. MNRAS, 424(Aug.), 2936-2942.

Sanna, A., Méndez, M., Altamirano, D., Belloni, T., Hiemstra, B., and Linares, M. 2014. Broad iron emission line and kilohertz quasi-periodic oscillations in the neutron star system 4U 1636-53. MNRAS, 440(June), 3275-3284.

Shakura, N. I., and Sunyaev, R. A. 1973. Black holes in binary systems. Observational appearance. A\&A, 24, 337-355.

Sobolewska, M. A., Papadakis, I. E., Done, C., and Malzac, J. 2011. Evidence for a change in the $\mathrm{X}$-ray radiation mechanism in the hard state of Galactic black holes. MNRAS, 417(Oct.), 280-288.

Steiner, J. F., Remillard, R. A., García, J. A., and McClintock, J. E. 2016. Stronger Reflection from Black Hole Accretion Disks in Soft X-Ray States. ApJ, 829(Oct.), L22.

Stella, L., and Vietri, M. 1998. Lense-Thirring Precession and Quasi-periodic Oscillations in Low-Mass X-Ray Binaries. ApJ, 492(Jan.), L59-L62.

Stella, L., and Vietri, M. 1999. kHz Quasiperiodic Oscillations in Low-Mass X-Ray Binaries as Probes of General Relativity in the Strong-Field Regime. Physical Review Letters, 82(Jan.), 17-20.

Stella, L., Vietri, M., and Morsink, S. M. 1999. Correlations in the Quasi-periodic Oscillation Frequencies of Low-Mass X-Ray Binaries and the Relativistic Precession Model. ApJ, 524(Oct.), L63-L66.

Stiele, H., Motta, S., Muñoz-Darias, T., and Belloni, T. M. 2011. Spectral properties of transitions between soft and hard states in GX 339-4. MNRAS, 418(Dec.), 1746-1752.

Strohmayera, T. E. 2001. Discovery of a $450 \mathrm{HZ}$ Quasi-periodic Oscillation from the Microquasar GRO J1655-40 with the Rossi X-Ray Timing Explorer. ApJ, $\mathbf{5 5 2 ( M a y ) , ~ L 4 9 - L 5 3 . ~}$

Strohmayerb, T. E. 2001. Discovery of a Second High-Frequency Quasi-periodic Oscillation from the Microquasar GRS 1915+105. ApJ, 554(June), L169L172.

Sunyaev, R., and Revnivtsev, M. 2000. Fourier power spectra at high frequencies: a way to distinguish a neutron star from a black hole. A\&A, 358(June), 617-623.

Takizawa, M., Dotani, T., Mitsuda, K., Matsuba, E., Ogawa, M., Aoki, T., Asai, K., Ebisawa, K., Makishima, K., Miyamoto, S., Iga, S., Vaughan, B., Rutledge, R. E., and Lewin, W. H. G. 1997. Spectral and Temporal Variability in the X-Ray Flux of GS 1124-683, Nova Muscae 1991. ApJ, 489(Nov.), 272-283.

Tananbaum, H., Gursky, H., Kellogg, E., Giacconi, R., and Jones, C. 1972. Observation of a Correlated X-Ray Transition in Cygnus X-1. ApJ, 177(Oct.), L5.

Titarchuk, L., and Fiorito, R. 2004. Spectral Index and Quasi-Periodic Oscillation Frequency Correlation in Black Hole Sources: Observational Evidence of Two Phases and Phase Transition in Black Holes. ApJ, 612(Sept.), 988-999.

Turolla, R., Zane, S., and Titarchuk, L. 2002. Power-Law Tails from Dynamical Comptonization in Converging Flows. ApJ, 576(Sept.), 349-356.

Uttley, P., Wilkinson, T., Cassatella, P., Wilms, J., Pottschmidt, K., Hanke, M., and Böck, M. 2011. The causal connection between disc and power-law variability in hard state black hole X-ray binaries. MNRAS, 414(June), L60-L64. 
van der Klis, M. 1994. Similarities in neutron star and black hole accretion. ApJS, 92(June), 511-519.

van der Klis, M. 2001. A Possible Explanation for the "Parallel Tracks" Phenomenon in Low-Mass X-Ray Binaries. ApJ, 561(Nov.), 943-949.

van der Klis, M. 2006 (Apr.). Rapid X-ray Variability. Pages 39-112 of: Lewin, W. H. G., and van der Klis, M. (eds), Compact stellar X-ray sources.

van Straaten, S., van der Klis, M., and Wijnands, R. 2005. Relations Between Timing Features and Colors in Accreting Millisecond Pulsars. ApJ, 619(Jan.), $455-482$.

Warner, B., Woudt, P. A., and Pretorius, M. L. 2003. Dwarf nova oscillations and quasi-periodic oscillations in cataclysmic variables - III. A new kind of dwarf nova oscillation, and further examples of the similarities to X-ray binaries. MNRAS, 344(Oct.), 1193-1209.

White, N. E., Stella, L., and Parmar, A. N. 1988. The X-ray spectral properties of accretion discs in X-ray binaries. ApJ, 324(Jan.), 363-378.

Wijnands, R., and van der Klis, M. 1999. The Broadband Power Spectra of X-Ray Binaries. ApJ, 514(Apr.), 939-944.

Wijnands, R., van der Klis, M., Homan, J., Chakrabarty, D., Markwardt, C. B., and Morgan, E. H. 2003. Quasi-periodic X-ray brightness fluctuations in an accreting millisecond pulsar. Nature, 424(July), 44-47.

Wijnands, R., Degenaar, N., Armas Padilla, M., Altamirano, D., Cavecchi, Y., Linares, M., Bahramian, A., and Heinke, C. O. 2015. Low-level accretion in neutron star X-ray binaries. MNRAS, 454(Dec.), 1371-1386.

Wilkinson, T., and Uttley, P. 2009. Accretion disc variability in the hard state of black hole X-ray binaries. MNRAS, 397(Aug.), 666-676.

Wilms, J., Nowak, M. A., Pottschmidt, K., Pooley, G. G., and Fritz, S. 2006. Long term variability of Cygnus X-1. IV. Spectral evolution 1999-2004. A\&A, 447(Feb.), 245-261.

Zdziarski, A. A., Lubiński, P., and Sikora, M. 2012. The MeV spectral tail in Cyg X-1 and optically thin emission of jets. MNRAS, 423(June), 663-675. 